


\title{
Thermo field hydrodynamic and kinetic equations of dense quantum nuclear systems
}

\author{
M.V.Tokarchuk ${ }^{1}$, T.Arimitsu $^{2}$, A.E.Kobryn ${ }^{1}$ \\ 1 Institute for Condensed Matter Physics of the Ukrainian National Academy \\ of Sciences, 1 Svientsitskii St., UA-290011 Lviv-11, Ukraine \\ 2 Institute of Physics, University of Tsukuba, Ibaraki 305, Japan
}

Received June 23, 1998

Basic equations of nonequilibrium thermo field dynamics of dense quantum systems are presented. A formulation of nonequilibrium thermo field dynamics has been performed using the nonequilibrium statistical operator method by D.N.Zubarev. Hydrodynamic equations have been obtained in thermo field representation. Two levels of the description of kinetics and hydrodynamics of a dense nuclear matter are considered. The first one is a quantum system with strongly coupled states, the second one is a quarkgluon plasma. Generalized transfer equations of a consistent description of kinetics and hydrodynamics have been obtained, as well as limiting cases are considered.

Key words: nonequilibrium thermo field dynamics, kinetics, hydrodynamics, kinetic equations, transport coefficients, coupled states, quark-gluon plasma

PACS: $12.38 . \mathrm{Mh}, 24.85 .+p, 52.25 . \mathrm{Dg}, 52.25 . \mathrm{Fi}, 82.20 . \mathrm{Mj}$

\section{Introduction}

The development of methods for the construction of kinetic and hydrodynamic equations in the theory of nonequilibrium processes for temperature quantum field systems is, in particular, important for the investigation of nonequilibrium properties of a quark-gluon plasma [1-5] - one of the nuclear matter states which can be created at ultrarelativistic collisions of heavy nuclei [6-9]. The description of strongly nonequilibrium processes of a nuclear matter appears also in the laser thermonuclear synthesis in systems D-D, D-T, D- ${ }^{3} \mathrm{He}, \mathrm{p}-\mathrm{B}[10-13]$. In theoretical investigations of the laser thermonuclear synthesis there is a problem of description of a laser beam propagation and its absorption by the target (in particular, by the D-T mixture), electron and photon energy transport in an ionized magnetized 
plasma with the creation of a corona - an electron liquid $\left(n_{e} \sim 10^{21} \div 10^{22} \mathrm{~cm}^{-3}\right)$, and the creation of a core - a super dense ion liquid $\left(n \sim 10^{24} \div 10^{26} \mathrm{~cm}^{-3}\right)$. In target ablation and implosion processes it is important to note transport coefficients, such as thermal conductivity, electron's conductivity, as well as dielectric properties, generation and evolution of spontaneous magnetic fields (with the inductivity of thousands of Tesla), nuclear synthesis mechanisms and energy transport by $\alpha$ particles and neutrons which are products of synthesis reactions. In this connection, there is a series of important problems, when kinetic and hydrodynamic processes in a magnetized super dense electron-ion plasma should be described consistently. Nonlinear hydrodynamic fluctuations of a magnetized degenerate electron liquid in a corona should be considered by taking into account both the kinetics of super heat electrons and nonlinear hydrodynamics of a super dense magnetized ion liquid, where nuclear synthesis reactions take place with the creation of high energy neutrons (with concentration $\sim 10^{24} \mathrm{~cm}^{-3}$ ) and $\alpha$-particles. The kinetics of these particles affects the synthesis processes and energy transport in the whole system. From the point of view of theoretical methods, in order to describe such strongly correlated nonequilibrium processes, one needs to construct the kinetics and hydrodynamics of a super dense high temperature electron-ion plasma and a nuclear matter at ultrarelativistic collisions of heavy nuclei. A nuclear matter - a stage of a quark-gluon plasma - appears after the compression of the target core of D-D, D-T plasma. As this takes place, its density increases by a factor of ten in the fourth degree and the distance between nuclons in the centre reaches $\sim 10^{-13} \mathrm{~cm}$. Such systems are examples of strong both long-range and short-range (nuclear) interactions. There is no small parameter for these systems (density, for example). Nonequilibrium processes have a strongly correlated collective nature. That is why methods which are based on a one-particle description, in particular, on the basis of Boltzmann-like kinetic equations, cannot be used. In addition to high temperature dense quantum systems, there are Bose and Fermi systems at low temperatures with decisive many-particle dissipative correlations. Neither the linear response theory nor Boltzmann-like kinetic equations are sufficient for their description.

Analysis of the problem of a description of kinetic processes in highly nonequilibrium and strongly coupled quantum systems on the basis of the nonequilibrium real-time Green functions technique [14-16] and the theory in terms of nonMarkovian kinetic equations describing memory effects [17-19] was made in recent paper [20] and then in monograph [21]. It is important to note that in [20] the quantum kinetic equation for a dense and strongly coupled nonequilibrium system was obtained when the parameters of a shortened description included a one-particle Wigner distribution function and an average energy density. On the basis of this approach the quantum Enskog kinetic equation was obtained in [21]. This equation is the quantum analogue of the classical one within the revised Enskog theory $[22,23]$. Problems of the construction of kinetic and hydrodynamic equations for highly nonequilibrium and strongly coupled quantum systems were considered based on the nonequilibrium thermo field dynamics in [24-29]. In par- 
ticular, a generalized kinetic equation for the average value of the Klimontovich operator was obtained in [25] with the help of the Kawasaki-Gunton projection operator method. The formalism of the nonequilibrium thermo field dynamics was applied to the description of a hydrodynamic state of quantum field systems in paper [26]. Generalized transport equations for nonequilibrium quantum systems, specifically for kinetic and hydrodynamic stages, were obtained in [27] on the basis of the thermo field dynamics conception [31,32] using the nonequilibrium statistical operator method $[21,33,34]$. In this approach, similarly to [20,21], the decisive role is that a set of the observed quantities is included in the description of the nonequilibrium process. For these quantities one finds generalized transport equations which should agree with nonequilibrium thermodynamics at controlling the local conservation laws for the particles-number density, momentum and energy. It gives substantial advantages over the nonequilibrium Green function technique [14-16], which quite well describes excitation spectra, but practically does not describe nonequilibrium thermodynamics, and has problems with the local conservation laws control and the generalized transport coefficients calculation.

In this paper we consider the kinetics and hydrodynamics of highly nonequilibrium and strongly coupled quantum systems using the nonequilibrium thermo field dynamics on the basis of the D.N.Zubarev nonequilibrium statistical operator method [27]. Within this method we consider two different levels of a description of the kinetics and hydrodynamics of dense quantum nuclear systems: strongly coupled states and a quark-gluon plasma. The nonequilibrium thermo field dynamics on the basis of the nonequilibrium statistical operator method constitutes sections 2 to 7 . A nonequilibrium thermo vacuum state vector is obtained here in view of equations for the generalized hydrodynamics of dense quantum systems. Transport equations of a consistent description of the kinetics and hydrodynamics in thermo field representation are obtained in section 8 . We mean that these equations are applied to dense quantum systems where strong coupled states can appear. This item implies, as one of the approaches, to investigate a nonequilibrium nuclear matter $[8,9]$. Another level of the description (rather a microscopic one) of nonequilibrium properties of dense quantum systems is considered in section 9. A consistent description of the kinetics and hydrodynamics is obtained here for a quark-gluon plasma.

\section{Thermo field dynamics formalism. Superoperators and state vectors in the Liouville thermo field space}

In this section we reformulate the nonequilibrium statistical mechanics of quantum systems using the thermo field dynamics formalism [31,32].

Let us consider a quantum system of $N$ interacting bosons or fermions. The Hamiltonian of this system is expressed via creation $a_{l}^{+}$and annihilation $a_{l}$ operators of the corresponding statistics:

$$
H=H\left(a^{+}, a\right)
$$


Operators $a_{l}^{+}, a_{l}$ satisfy the commutation relations:

$$
\left[a_{l}, a_{j}^{+}\right]_{\sigma}=\delta_{l j}, \quad\left[a_{l}, a_{j}\right]_{\sigma}=\left[a_{l}^{+}, a_{j}^{+}\right]_{\sigma}=0,
$$

where $[A, B]_{\sigma}=A B-\sigma B A, \sigma=+1$ for bosons and $\sigma=-1$ for fermions.

The nonequilibrium state of such a system is completely described by the nonequilibrium statistical operator $\varrho(t)$. This operator satisfies the quantum Liouville equation

$$
\frac{\partial}{\partial t} \varrho(t)-\frac{1}{\mathrm{i} \hbar}[H, \varrho(t)]=0
$$

The nonequilibrium statistical operator $\varrho(t)$ allows us to calculate the average values of operators $A$

$$
\langle A\rangle^{t}=\operatorname{Sp}(A \varrho(t)),
$$

which can be observable quantities describing the nonequilibrium state of the system (for example, a hydrodynamic state is described by the average values of operators of particle number, momentum and energy densities).

The main idea of thermo field dynamics [31,32] and its nonequilibrium formulation [35-37] consists in doing the calculation of average values (2.4) with the help of the so-called nonequilibrium thermo vacuum state vector:

$$
\langle A\rangle^{t}=\langle\langle 1 \mid A \varrho(t)\rangle\rangle=\langle\langle 1|\hat{A}| \varrho(t)\rangle\rangle,
$$

where $\hat{A}$ is a superoperator which acts on state $|\varrho(t)\rangle\rangle$. Nonequilibrium thermo vacuum state vector $|\varrho(t)\rangle\rangle$ satisfies the Schrödinger equation. Starting from equation $(2.3)$, we obtain the relation

$$
\left.\left.\frac{\partial}{\partial t}|\varrho(t)\rangle\right\rangle-\left|\frac{1}{\mathrm{i} \hbar}[H, \varrho(t)]\right\rangle\right\rangle=0
$$

or, opening commutator,

$$
\left.\left.\frac{\partial}{\partial t}|\varrho(t)\rangle\right\rangle-\frac{1}{\mathrm{i} \hbar} \bar{H}|\varrho(t)\rangle\right\rangle=0 .
$$

Here the "total" Hamiltonian $\bar{H}$ reads:

$$
\bar{H}=\hat{H}-\tilde{H},
$$

and it is known that $\left\langle\langle 1| \bar{H}=0 ; \hat{H}=H\left(\hat{a}^{+}, \hat{a}\right), \tilde{H}=H^{*}\left(\tilde{a}^{+}, \tilde{a}\right)\right.$ are superoperators which consist of creation and annihilation superoperators without and with a tilde, and which represent the thermal Liouville space [38,39]. Superoperators $\hat{H}$ and $\tilde{H}$ are defined in accordance with the relations:

$$
\begin{aligned}
|H \varrho(t)\rangle\rangle & =\hat{H}|\varrho(t)\rangle\rangle, \\
|\varrho(t) H\rangle\rangle & =\tilde{H}|\varrho(t)\rangle\rangle .
\end{aligned}
$$


Hence, it appears that at going from the quantum Liouville equation (2.3) for nonequilibrium statistical operator $\varrho(t)$ to the Schrödinger equation (2.6) for nonequilibrium thermo vacuum state vector $|\varrho(t)\rangle\rangle$, according to (2.5), the number of creation and annihilation operators is doubled. Superoperators $\hat{a}_{l}^{+}, \hat{a}_{j}, \tilde{a}_{l}^{+}, \tilde{a}_{j}$ satisfy the same commutation relations as for operators $a_{l}^{+}, a_{j}(2.2)$ :

$$
\begin{array}{ll}
{\left[\hat{a}_{l}, \hat{a}_{j}^{+}\right]_{\sigma}=\left[\tilde{a}_{l}, \tilde{a}_{j}^{+}\right]_{\sigma}=\delta_{l j},} & {\left[\hat{a}_{l}, \tilde{a}_{j}\right]_{\sigma}=\left[\hat{a}_{l}^{+}, \tilde{a}_{j}^{+}\right]_{\sigma}=0,} \\
{\left[\hat{a}_{l}, \hat{a}_{j}\right]_{\sigma}=\left[\hat{a}_{l}^{+}, \hat{a}_{j}^{+}\right]_{\sigma}=0,} & {\left[\tilde{a}_{l}, \tilde{a}_{j}\right]_{\sigma}=\left[\tilde{a}_{l}^{+}, \tilde{a}_{j}^{+}\right]_{\sigma}=0 .}
\end{array}
$$

Annihilation superoperators $\hat{a}_{l}, \tilde{a}_{l}$ are defined in accordance with their action on the vacuum state - the supervacuum [38]

$$
\left.\left.\hat{a}_{l}|00\rangle\right\rangle=\tilde{a}_{l}|00\rangle\right\rangle=0,
$$

where $|00\rangle\rangle=\| 0\rangle\langle 0 \mid\rangle\rangle$ is a supervacuum; and it is known that $\hat{a}_{l}|0\rangle=a_{l}|0\rangle=0$, and $\langle 0| \tilde{a}_{l}=0$, i.e. a supervacuum $\left.|00\rangle\right\rangle$ is an orthogonalized state of two vacuum states $\langle 0|$ and $|0\rangle$. Taking into account commutation relations (2.9), (2.10), one can introduce unit vectors $\left.\left.|1\rangle\rangle=\left|\sum_{l}\right| l\right\rangle\langle l \mid\rangle\right\rangle$ and $\left\langle\langle 1|=\left\langle\left\langle\sum_{l} \mid l\right\rangle\langle l||\right.\right.$ in the following forms:

$$
\begin{aligned}
& \left.|1\rangle\rangle=\exp \left\{\sum_{l} \hat{a}_{l}^{+} \tilde{a}_{l}^{+}\right\}|00\rangle\right\rangle, \\
& \left\langle\langle 1|=\left\langle\langle 00| \exp \left\{\sum_{l} \tilde{a}_{l} \hat{a}_{l}\right\} .\right.\right.
\end{aligned}
$$

With the help of these expressions one can find relations between the action of superoperators $\hat{a}_{l}^{+}, \hat{a}_{j}, \tilde{a}_{l}^{+}, \tilde{a}_{j}$

$$
\begin{array}{ll}
\left.\left.\hat{a}_{l}|1\rangle\right\rangle=\tilde{a}_{l}^{+}|1\rangle\right\rangle, \quad & \left\langle\langle 1| \hat{a}_{l}^{+}=\left\langle\langle 1| \tilde{a}_{l},\right.\right. \\
\left.\left.\hat{a}_{l}^{+}|1\rangle\right\rangle=\sigma \tilde{a}_{l}|1\rangle\right\rangle, & \left\langle\langle 1| \hat{a}_{l}=\left\langle\langle 1| \tilde{a}_{l}^{+} \sigma .\right.\right.
\end{array}
$$

In such a way, in the thermal field dynamics formalism $[31,32]$ the number of operators is doubled by introducing both without tilde and tildian operators $A\left(\hat{a}^{+}, \hat{a}\right)$, $\tilde{A}\left(\tilde{a}^{+}, \tilde{a}\right)$ for which the following properties take place:

$$
\begin{aligned}
\widetilde{A_{1} A_{2}} & =\tilde{A}_{1} \tilde{A}_{2}, \quad \tilde{\tilde{A}}=A, \\
c_{1} A_{1}+c_{2} A_{2} & =c_{1}^{*} \tilde{A}_{1}+c_{2}^{*} \tilde{A}_{2}, \\
|A\rangle\rangle & =\hat{A}|1\rangle\rangle, \\
\left.\left|A_{1} A_{2}\right\rangle\right\rangle & \left.=\hat{A}_{1}\left|A_{2}\right\rangle\right\rangle .
\end{aligned}
$$

Here $^{*}$ denotes a complex conjugation. Some detailed description of the properties of superoperators $\hat{a}_{l}^{+}, \hat{a}_{j}, \tilde{a}_{l}^{+}, \tilde{a}_{j}$, as well as a thermal Liouville space is given in papers $[31,32,38,39]$.

The nonequilibrium thermo vacuum state vector is normalized

$$
\langle\langle 1 \mid \varrho(t)\rangle\rangle=\langle\langle 1|\hat{\varrho}(t)| 1\rangle\rangle=1,
$$

where $\hat{\varrho}(t)$ is a nonequilibrium statistical superoperator. It depends on superoperators $a_{l}^{+}, a_{l}$

$$
\hat{\varrho}(t) \equiv \varrho\left(\hat{a}^{+}, \hat{a} ; t\right),
$$

and, it is known that the corresponding tildian superoperator $\tilde{\varrho}(t) \equiv \varrho^{+}\left(\tilde{a}^{+}, \tilde{a} ; t\right)$ depends on superoperators $\tilde{a}_{l}^{+}, \tilde{a}_{l}$. 


\section{Nonequilibrium statistical operator in thermo field space}

To solve the Schrödinger equation (2.6) a boundary condition should be given. Following the nonequilibrium statistical operator method [21,27,33,34], let us find a solution to this equation in a form, which depends on time via some set of observable quantities only. It means that this set is sufficient for the description of a nonequilibrium state of a system and does not depend on the initial moment of time. The solution to the Schrödinger equation, which satisfies the following boundary condition

$$
\left.|\varrho(t)\rangle\rangle_{t=t_{0}}=\left|\varrho_{\mathrm{q}}\left(t_{0}\right)\right\rangle\right\rangle,
$$

reads:

$$
\left.|\varrho(t)\rangle\rangle=\exp \left\{\left(t-t_{0}\right) \frac{1}{\mathrm{i} \hbar} \bar{H}\right\}\left|\varrho_{\mathrm{q}}\left(t_{0}\right)\right\rangle\right\rangle .
$$

We will consider times $t \gg t_{0}$, when the details of the initial state become inessential. To avoid the dependence on $t_{0}$, let us average the solution (3.2) on the initial time moment in the range between $t_{0}$ and $t$ and make the limiting transition $t_{0}-t \rightarrow-\infty$. We will obtain [27]:

$$
\left.|\varrho(t)\rangle\rangle=\varepsilon \int_{-\infty}^{0} \mathrm{~d} t^{\prime} \mathrm{e}^{\varepsilon t^{\prime}} \mathrm{e}^{-\frac{1}{\mathrm{i} \hbar} \bar{H} t}\left|\varrho_{\mathrm{q}}\left(t+t^{\prime}\right)\right\rangle\right\rangle,
$$

where $\varepsilon \rightarrow+0$ after the thermodynamic limiting transition. Solution (3.3), as it can be shown by its direct differentiation with respect to time $t$, satisfies the Schrödinger equation with a small source in the right-hand side:

$$
\left.\left.\left.\left(\frac{\partial}{\partial t}-\frac{1}{\mathrm{i} \hbar} \bar{H}\right)|\varrho(t)\rangle\right\rangle=-\varepsilon(|\varrho(t)\rangle\rangle-\left|\varrho_{\mathrm{q}}(t)\right\rangle\right\rangle\right) .
$$

This source selects retarded solutions which correspond to a shortened description of the nonequilibrium state of a system, $\left.\left|\varrho_{\mathrm{q}}(t)\right\rangle\right\rangle$ is a thermo vacuum quasiequilibrium state vector

$$
\left.\left.\left|\varrho_{\mathrm{q}}(t)\right\rangle\right\rangle=\hat{\varrho}_{\mathrm{q}}(t)|1\rangle\right\rangle .
$$

Similarly to (2.14), it is normalized by the rule

$$
\left\langle\left\langle 1 \mid \varrho_{\mathrm{q}}(t)\right\rangle\right\rangle=\left\langle\left\langle 1\left|\hat{\varrho}_{\mathrm{q}}(t)\right| 1\right\rangle\right\rangle=1,
$$

where $\hat{\varrho}_{q}(t)$ is a quasiequilibrium statistical superoperator. The quasiequilibrium thermo vacuum state vector of a system is introduced in the following way. Let $\left\langle p_{n}\right\rangle^{t}=\left\langle\left\langle 1\left|\hat{p}_{n}\right| \varrho(t)\right\rangle\right\rangle$ be a set of observable quantities which describe the nonequilibrium state of a system. $p_{n}$ are operators which consist of the creation and annihilation operators defined in (2.2). Quasiequilibrium statistical operator $\varrho_{\mathrm{q}}(t)$ is defined from the condition of informational entropy $S_{\text {inf }}$ extremum (maximum) at 
additional conditions of prescribing the average values $\left\langle p_{n}\right\rangle^{t}$ and conservation of normalization condition (3.6) [21,34]:

$$
\begin{aligned}
& \varrho_{\mathrm{q}}(t)=\exp \left\{-\Phi(t)-\sum_{n} F_{n}^{*}(t) p_{n}\right\}, \\
& \Phi(t)=\ln \operatorname{Sp} \exp \left\{-\sum_{n} F_{n}^{*}(t) p_{n}\right\},
\end{aligned}
$$

where $\Phi(t)$ is the Massieu-Planck functional. A summation on $n$ can designate a sum with respect to the wave-vector $\boldsymbol{k}$, the kind of particles and a line of other quantum numbers, a spin for example. Parameters $F_{n}(t)$ are defined from the conditions of self-consistency:

$$
\left\langle p_{n}\right\rangle^{t}=\left\langle p_{n}\right\rangle_{\mathrm{q}}^{t}, \quad\langle\ldots\rangle_{\mathrm{q}}^{t}=\operatorname{Sp}\left(\ldots \varrho_{\mathrm{q}}(t)\right) .
$$

According to (2.5), let us write these conditions of self-consistency in the following form:

$$
\left\langle\left\langle 1\left|\hat{p}_{n}\right| \varrho(t)\right\rangle\right\rangle=\left\langle\left\langle 1\left|\hat{p}_{n}\right| \varrho_{\mathrm{q}}(t)\right\rangle\right\rangle .
$$

Taking into account behaviours (2.13), we have:

$$
\left.\left.\left.\left|\varrho_{\mathrm{q}}(t)\right\rangle\right\rangle=\hat{\varrho}_{\mathrm{q}}(t)|1\rangle\right\rangle=\tilde{\varrho}_{\mathrm{q}}^{+}(t)|1\rangle\right\rangle,
$$

where

$$
\begin{aligned}
& \hat{\varrho}_{\mathrm{q}}(t)=\exp \left\{-\Phi(t)-\sum_{n} F_{n}^{*}(t) \hat{p}_{n}\right\}, \\
& \tilde{\varrho}_{\mathrm{q}}^{+}(t)=\exp \left\{-\Phi(t)-\sum_{n} F_{n}(t) \tilde{p}_{n}\right\}
\end{aligned}
$$

are quasiequilibrium statistical superoperators which contain superoperators $\hat{p}_{n}$ and $\tilde{p}_{n}$, correspondingly:

$$
\begin{aligned}
& \hat{p}_{n}=p_{n}\left(\hat{a}^{+}, \hat{a}\right), \\
& \tilde{p}_{n}=p_{n}^{*}\left(\tilde{a}^{+}, \tilde{a}\right) .
\end{aligned}
$$

If self-consistency condition (3.9) realizes, we shall have the following relations (at fixed corresponding parameters):

$$
\begin{aligned}
& \frac{\delta \Phi(t)}{\delta F_{n}^{*}(t)}=\left\langle\left\langle 1\left|\hat{p}_{n}\right| \varrho_{\mathrm{q}}(t)\right\rangle\right\rangle=\left\langle\left\langle 1\left|\hat{p}_{n}\right| \varrho(t)\right\rangle\right\rangle, \\
& \frac{\delta \Phi(t)}{\delta F_{n}(t)}=\left\langle\left\langle 1\left|\tilde{p}_{n}\right| \varrho_{\mathrm{q}}(t)\right\rangle\right\rangle=\left\langle\left\langle 1\left|\tilde{p}_{n}\right| \varrho(t)\right\rangle\right\rangle .
\end{aligned}
$$


Relations (3.13) show that parameters $F_{n}^{*}(t), F_{n}(t)$ are conjugated to averages $\left\langle\left\langle 1\left|\hat{p}_{n}\right| \varrho(t)\right\rangle\right\rangle$ and $\left\langle\left\langle 1\left|\tilde{p}_{n}\right| \varrho(t)\right\rangle\right\rangle$, correspondingly. On the other hand, with the help of $\left.\left|\varrho_{\mathrm{q}}(t)\right\rangle\right\rangle$ and self-consistency conditions (3.9) we can define the entropy of the system state:

$$
S(t)=-\left\langle\left\langle 1 \mid\left(\ln \varrho_{\mathrm{q}}(t)\right) \varrho_{\mathrm{q}}(t)\right\rangle\right\rangle=\Phi(t)+\sum_{n} F_{n}^{*}(t)\left\langle\left\langle 1\left|\hat{p}_{n}\right| \varrho(t)\right\rangle\right\rangle .
$$

The physical meaning of parameters $F_{n}^{*}(t)$ can be obtained now on the basis of the previous relation:

$$
F_{n}^{*}(t)=\frac{\delta S(t)}{\delta\left\langle\left\langle 1\left|\hat{p}_{n}\right| \varrho(t)\right.\right.} .
$$

\section{Projection operators in thermo field space}

Now the auxiliary quasiequilibrium thermo vacuum state vector $\left.\left|\varrho_{\mathrm{q}}(t)\right\rangle\right\rangle$ is defined. Let us represent solution (3.3) of the Schrödinger equation (2.6) in a form which is more convenient for the construction of transport equations for averages $\left\langle\left\langle 1\left|\hat{p}_{n}\right| \varrho(t)\right\rangle\right\rangle$. We shall start from the Schrödinger equation with a small source (3.4). Let us rebuild this equation by introducing $\left.\Delta|\varrho(t)\rangle\rangle=|\varrho(t)\rangle\rangle-\left|\varrho_{\mathrm{q}}(t)\right\rangle\right\rangle$ :

$$
\left.\left.\left(\frac{\partial}{\partial t}-\frac{1}{\mathrm{i} \hbar} \bar{H}+\varepsilon\right) \Delta|\varrho(t)\rangle\right\rangle=-\left(\frac{\partial}{\partial t}-\frac{1}{\mathrm{i} \hbar} \bar{H}\right)\left|\varrho_{\mathrm{q}}(t)\right\rangle\right\rangle .
$$

The calculation of time derivation of $\left.\left|\varrho_{\mathrm{q}}(t)\right\rangle\right\rangle$ in the right-hand side of equation (4.1) is equivalent to the introduction of the Kawasaki-Gunton projection operator $\mathscr{P}_{\mathrm{q}}(t)[27]$ in thermo field representation:

$$
\begin{aligned}
& \left.\left.\frac{\partial}{\partial t}\left|\varrho_{\mathrm{q}}(t)\right\rangle\right\rangle=\mathscr{P}_{\mathrm{q}}(t) \frac{1}{\mathrm{i} \hbar} \bar{H}|\varrho(t)\rangle\right\rangle, \\
& \left.\left.\mathscr{P}_{\mathrm{q}}(t)(|\ldots\rangle\rangle\right)=\left|\varrho_{\mathrm{q}}(t)\right\rangle\right\rangle+ \\
& \sum_{n} \frac{\left.\delta\left|\varrho_{\mathrm{q}}(t)\right\rangle\right\rangle}{\delta\left\langle\left\langle 1\left|\hat{p}_{n}\right| \varrho(t)\right\rangle\right\rangle}\left\langle\left\langle 1\left|\hat{p}_{n}\right| \ldots\right\rangle\right\rangle-\sum_{n} \frac{\left.\delta\left|\varrho_{\mathrm{q}}(t)\right\rangle\right\rangle}{\delta\left\langle\left\langle 1\left|\hat{p}_{n}\right| \varrho(t)\right\rangle\right\rangle}\left\langle\left\langle 1\left|\hat{p}_{n}\right| \ldots\right\rangle\right\rangle\langle\langle 1 \mid \ldots\rangle\rangle .
\end{aligned}
$$

Projection operator $\mathscr{P}_{\mathrm{q}}(t)$ acts on state vectors $\left.|\ldots\rangle\right\rangle$ only and has all the operator properties:

$$
\begin{aligned}
& \left.\left.\mathscr{P}_{\mathrm{q}}(t)\left|\varrho\left(t^{\prime}\right)\right\rangle\right\rangle=\left|\varrho_{\mathrm{q}}(t)\right\rangle\right\rangle, \\
& \left.\left.\mathscr{P}_{\mathrm{q}}(t)\left|\varrho_{\mathrm{q}}\left(t^{\prime}\right)\right\rangle\right\rangle=\left|\varrho_{\mathrm{q}}(t)\right\rangle\right\rangle, \\
& \mathscr{P}_{\mathrm{q}}(t) \mathscr{P}_{\mathrm{q}}\left(t^{\prime}\right)=\mathscr{P}_{\mathrm{q}}(t) .
\end{aligned}
$$

Taking into account condition $\left.\mathscr{P}_{\mathrm{q}}(t) \frac{1}{\mathrm{i} \hbar} \bar{H} \Delta|\varrho(t)\rangle\right\rangle=0$, one may rewrite equation (4.1), after simple reductions, in a form:

$$
\left.\left.\left(\frac{\partial}{\partial t}-\left(1-\mathscr{P}_{\mathrm{q}}(t)\right) \frac{1}{\mathrm{i} \hbar} \bar{H}+\varepsilon\right) \Delta|\varrho(t)\rangle\right\rangle=\left(1-\mathscr{P}_{\mathrm{q}}(t)\right) \frac{1}{\mathrm{i} \hbar} \bar{H}\left|\varrho_{\mathrm{q}}(t)\right\rangle\right\rangle .
$$


The formal solution to this equation reads:

$$
\left.\Delta|\varrho(t)\rangle\rangle=\int_{-\infty}^{t} \mathrm{~d} t^{\prime} \mathrm{e}^{\varepsilon\left(t^{\prime}-t\right)} T\left(t, t^{\prime}\right)\left(1-\mathscr{P}_{\mathrm{q}}\left(t^{\prime}\right)\right) \frac{1}{\mathrm{i} \hbar} \bar{H}\left|\varrho_{\mathrm{q}}\left(t^{\prime}\right)\right\rangle\right\rangle
$$

or

$$
\left.\left.|\varrho(t)\rangle\rangle=\left|\varrho_{\mathrm{q}}(t)\right\rangle\right\rangle+\int_{-\infty}^{t} \mathrm{~d} t^{\prime} \mathrm{e}^{\varepsilon\left(t^{\prime}-t\right)} T\left(t, t^{\prime}\right)\left(1-\mathscr{P}_{\mathrm{q}}\left(t^{\prime}\right)\right) \frac{1}{\mathrm{i} \hbar} \bar{H}\left|\varrho_{\mathrm{q}}\left(t^{\prime}\right)\right\rangle\right\rangle,
$$

where

$$
T\left(t, t^{\prime}\right)=\exp _{+}\left\{\int_{t^{\prime}}^{t} \mathrm{~d} t^{\prime}\left(1-\mathscr{P}_{\mathrm{q}}\left(t^{\prime}\right)\right) \frac{1}{\mathrm{i} \hbar} \bar{H}\right\}
$$

is an evolution operator with projection consideration, and $\exp _{+}$is an ordered exponent. Then, let us consider expression $\left.\left(1-\mathscr{P}_{\mathrm{q}}\left(t^{\prime}\right)\right) \frac{1}{\mathrm{i} \hbar} \bar{H}\left|\varrho_{\mathrm{q}}(t)\right\rangle\right\rangle$ in the righthand side of (4.1). The action of $\frac{1}{\mathrm{i} \hbar} \bar{H}$ and $\left(1-\mathscr{P}_{\mathrm{q}}\left(t^{\prime}\right)\right)$ on $\left.\left|\varrho_{\mathrm{q}}(t)\right\rangle\right\rangle$ can be represented in the form:

$$
\left.\left.\left(1-\mathscr{P}_{\mathrm{q}}\left(t^{\prime}\right)\right) \frac{1}{\mathrm{i} \hbar} \bar{H}\left|\varrho_{\mathrm{q}}(t)\right\rangle\right\rangle=\sum_{n} F_{n}^{*}(t)\left|\int_{0}^{1} \mathrm{~d} \tau \varrho_{\mathrm{q}}^{\tau}(t)\left(1-\mathscr{P}\left(t^{\prime}\right)\right) \dot{p}_{n} \varrho_{\mathrm{q}}^{1-\tau}(t)\right\rangle\right\rangle,(4
$$

where $\dot{p}_{n}$ and $\mathscr{P}(t)$ read:

$$
\begin{aligned}
\dot{p}_{n} & =-\frac{1}{\mathrm{i} \hbar}\left[H, p_{n}\right], \\
\mathscr{P}(t) p & =\left\langle\left\langle 1|\hat{p}| \varrho_{\mathrm{q}}(t)\right\rangle\right\rangle+\sum_{n} \frac{\delta\left\langle\left\langle 1|\hat{p}| \varrho_{\mathrm{q}}(t)\right\rangle\right\rangle}{\delta\left\langle\left\langle 1\left|\hat{p}_{n}\right| \varrho(t)\right\rangle\right\rangle}\left(p_{n}-\left\langle\left\langle 1\left|\hat{p}_{n}\right| \varrho(t)\right\rangle\right\rangle\right) .
\end{aligned}
$$

Here $\mathscr{P}(t)$ is a generalized Mori projection operator in thermo field representation. It acts on operators and has the following properties:

$$
\begin{array}{ll}
\mathscr{P}(t) p_{n} & =p_{n}, \\
\mathscr{P}(t) \mathscr{P}\left(t^{\prime}\right) & =\mathscr{P}(t) .
\end{array}
$$

Let us substitute now (4.7) into (4.5) and, as a result, we will obtain an expression for the nonequilibrium thermo vacuum state of a system:

$$
\left.\left.|\varrho(t)\rangle\rangle=\left|\varrho_{\mathrm{q}}(t)\right\rangle\right\rangle+\sum_{n} \int_{-\infty}^{t} \mathrm{~d} t^{\prime} \mathrm{e}^{\varepsilon\left(t^{\prime}-t\right)} T\left(t, t^{\prime}\right)\left|\int_{0}^{1} \mathrm{~d} \tau \varrho_{\mathrm{q}}^{\tau}\left(t^{\prime}\right) J_{n}\left(t^{\prime}\right) \varrho_{\mathrm{q}}^{1-\tau}\left(t^{\prime}\right)\right\rangle\right\rangle F_{n}^{*}\left(t^{\prime}\right) .
$$

Here

$$
J_{n}(t)=(1-\mathscr{P}(t)) \dot{p}_{n}
$$

are generalized flows. 
Let us obtain now transport equations for averages $\left\langle\left\langle 1\left|\hat{p}_{n}\right| \varrho(t)\right\rangle\right\rangle$ in thermo field representation with the help of nonequilibrium thermo vacuum state vector $|\varrho(t)\rangle\rangle$ (4.11). To achieve this we will use the equality

$$
\frac{\partial}{\partial t}\left\langle\left\langle 1\left|\hat{p}_{n}\right| \varrho(t)\right\rangle\right\rangle=\left\langle\left\langle 1\left|\dot{\hat{p}}_{n}\right| \varrho(t)\right\rangle\right\rangle=\left\langle\left\langle 1\left|\dot{\hat{p}}_{n}\right| \varrho_{\mathrm{q}}(t)\right\rangle\right\rangle+\left\langle\left\langle J_{n}(t) \mid \varrho(t)\right\rangle\right\rangle .
$$

By making use of $|\varrho(t)\rangle\rangle$ in (4.11) in averaging the last term, we obtain transport equations for $\left\langle\left\langle 1\left|\hat{p}_{n}\right| \varrho(t)\right\rangle\right\rangle$ :

$$
\begin{aligned}
& \frac{\partial}{\partial t}\left\langle\left\langle 1\left|\hat{p}_{n}\right| \varrho(t)\right\rangle\right\rangle=\left\langle\left\langle 1\left|\dot{\hat{p}}_{n}\right| \varrho_{\mathrm{q}}(t)\right\rangle\right\rangle+ \\
& \sum_{n^{\prime}} \int_{-\infty}^{t} \mathrm{~d} t^{\prime} \mathrm{e}^{\varepsilon\left(t^{\prime}-t\right)}\left\langle\left\langle J_{n}(t) T\left(t, t^{\prime}\right) \mid \int_{0}^{1} \mathrm{~d} \tau \varrho_{\mathrm{q}}^{\tau}\left(t^{\prime}\right) J_{n^{\prime}}\left(t^{\prime}\right) \varrho_{\mathrm{q}}^{1-\tau}\left(t^{\prime}\right)\right\rangle\right\rangle F_{n^{\prime}}^{*}\left(t^{\prime}\right),
\end{aligned}
$$

where $\dot{\hat{p}}_{n}=-\frac{1}{\mathrm{i} \hbar} \bar{H} \hat{p}_{n}$. Relations (4.14) are treated as a general form of transport equations for average values of a shortened description. These equations can be applied to completely actual problems.

In the case of weakly nonequilibrium processes, the generalized transport equations (4.14) are reduced appreciably. We shall consider this case in the next section.

\section{Transport equations in linear approximation}

Let us consider the nonequilibrium state of a quantum field system near equilibrium. In this connection let us suppose that average values $\left\langle\left\langle 1\left|\hat{p}_{n}\right| \varrho(t)\right\rangle\right\rangle$ of variables for a shortened description and their conjugated parameters $F_{n}^{*}(t)$ differ in their equilibrium meanings slightly. In such a case, one can expand the quasiequilibrium thermo vacuum state vector $\left.\left|\varrho_{\mathrm{q}}\left(t_{0}\right)\right\rangle\right\rangle(3.5)$, (3.11) into a series on deviations of parameters $F_{n}^{*}(t)$ from their equilibrium values $F_{n}(0)$ and restrict a linear approximation only:

$$
\left.\left.\left.\left|\varrho_{\mathrm{q}}(t)\right\rangle\right\rangle=\left|\varrho_{0}\left(F_{n}(0)\right)\right\rangle\right\rangle-\sum_{n} \delta F_{n}^{*}(t)\left|\int_{0}^{1} \mathrm{~d} \tau \varrho_{0}^{\tau}\left(F_{n}(0)\right) p_{n} \varrho_{0}^{1-\tau}\left(F_{n}(0)\right)\right\rangle\right\rangle,
$$

where $\left.\left|\varrho_{0}\left(F_{n}(0)\right)\right\rangle\right\rangle$ is an equilibrium thermo vacuum state vector which depends on equilibrium values of $F_{n}(0)$ parameters (local inversed temperature $\beta$ and chemical potential $\mu), \delta F_{n}^{*}(t)=F_{n}^{*}(t)-F_{n}^{*}(0)$. With the help of self-consistency conditions (3.9) and taking into account (5.1), let us define parameters $\delta F_{n}^{*}(t)$ :

$$
\delta F_{n}^{*}(t)=-\sum_{m}((p \mid p))_{n m}^{-1}\left\langle\left\langle 1\left|\delta \hat{p}_{m}\right| \varrho(t)\right\rangle\right\rangle
$$

where $\delta \hat{p}_{m}=\hat{p}_{m}-\left\langle\left\langle 1\left|\hat{p}_{m}\right| \varrho_{0}\left(F_{n}(0)\right)\right\rangle\right\rangle,((p \mid p))_{m n}^{-1}$ are elements of the inverse matrix of $((p \mid p))$. Elements of the matrix $((p \mid p))$ are equilibrium correlation functions in thermo field representation

$$
\left(\left(p_{n} \mid p_{m}\right)\right)_{0}=\left\langle\left\langle p_{n} \mid \int_{0}^{1} \mathrm{~d} \tau \varrho_{0}^{\tau} p_{m} \varrho_{0}^{1-\tau}\right\rangle\right\rangle .
$$


Substitution of (5.3) into (5.1) for $\left.\left|\varrho_{\mathrm{q}}(t)\right\rangle\right\rangle$ results in

$$
\left.\left.\left.\left|\varrho_{\mathrm{q}}(t)\right\rangle\right\rangle=\left|\varrho_{0}(F(0))\right\rangle\right\rangle+\sum_{m, n}\left\langle\left\langle 1\left|\delta \hat{p}_{m}\right| \varrho(t)\right\rangle\right\rangle((p \mid p))_{m n}^{-1}\left|\int_{0}^{1} \mathrm{~d} \tau \varrho_{0}^{\tau}(F(0)) p_{n} \varrho_{0}^{1-\tau}(F(0))\right\rangle\right\rangle .
$$

To calculate nonequilibrium thermo vacuum state vector $|\varrho(t)\rangle\rangle(4.11)$ in linear approximation (5.4), let us rewrite transport equations (4.14) in another form:

$$
\frac{\partial}{\partial t}\left\langle\left\langle 1\left|\delta \hat{p}_{n}\right| \varrho(t)\right\rangle\right\rangle=\sum_{m} \Omega_{n m}\left\langle\left\langle 1\left|\delta \hat{p}_{n}\right| \varrho(t)\right\rangle\right\rangle-\sum_{m} \int_{-\infty}^{t} \mathrm{~d} t^{\prime} \mathrm{e}^{\varepsilon\left(t^{\prime}-t\right)} \varphi_{n m}\left(t, t^{\prime}\right)\left\langle\left\langle 1\left|\delta \hat{p}_{n}\right| \varrho\left(t^{\prime}\right)\right\rangle\right\rangle,
$$

where

$$
\Omega_{n m}=\sum_{l}\left\langle\left\langle\dot{p}_{n} \mid \int_{0}^{1} \mathrm{~d} \tau \varrho_{0}^{\tau} p_{l} \varrho_{0}^{1-\tau}\right\rangle\right\rangle((p \mid p))_{l m}^{-1}
$$

are equilibrium quantum correlation functions, and

$$
\varphi_{n m}\left(t, t^{\prime}\right)=\sum_{l}\left\langle\left\langle J_{n}\left|T_{0}\left(t, t^{\prime}\right)\right| \int_{0}^{1} \mathrm{~d} \tau \varrho_{0}^{\tau} J_{l} \varrho_{0}^{1-\tau}\right\rangle\right\rangle((p \mid p))_{l m}^{-1}
$$

are transport cores in thermo field representation, which describe dissipative processes in the weakly nonequilibrium state of a quantum system, $J_{n}$ is defined similarly to (4.12):

$$
\begin{aligned}
J_{n} & =\left(1-\mathscr{P}_{0}\right) \dot{p}_{n} ; \\
T_{0}(t) & =\exp \left\{t\left(1-\mathscr{P}_{0}\right) \frac{\mathrm{i}}{\hbar} \bar{H}\right\}
\end{aligned}
$$

is the time evolution operator with taking into account the projecting where $\mathscr{P}_{0}$ is the Mori projection operator with the following structure:

$$
\mathscr{P}_{0} A=\left\langle\left\langle 1|\hat{A}| \varrho_{0}\right\rangle\right\rangle+\sum_{m, n}\left(\left(A \mid p_{n}\right)\right)((p \mid p))_{n m}^{-1} p_{m}
$$

Projection operator $\mathscr{P}_{0}$ satisfies conditions (4.10). As it can be shown [27], for transport equations (5.5) correspond to equations for quantum time correlation functions in thermo field representation:

$$
\begin{aligned}
\Phi_{n m}(t) & =\left\langle\left\langle p_{n}(t) \mid p_{m}(0) \varrho_{0}\right\rangle\right\rangle \\
\frac{\partial}{\partial t} \Phi_{n m}(t) & =\sum_{l} \Omega_{n l} \Phi_{l m}(t)-\sum_{l} \int_{-\infty}^{t} \mathrm{~d} t^{\prime} \mathrm{e}^{\varepsilon\left(t^{\prime}-t\right)} \varphi_{n l}\left(t, t^{\prime}\right) \Phi_{l m}\left(t^{\prime}\right),
\end{aligned}
$$

where the time evolution of superoperators $\hat{p}_{n}(t)$ in the Heisenberg representation reads:

$$
\hat{p}_{n}(t)=\exp \left\{-\frac{1}{\mathrm{i} \hbar} \bar{H} t\right\} \hat{p}_{n} \exp \left\{\frac{1}{\mathrm{i} \hbar} \bar{H} t\right\} .
$$


As it is indicated by (5.5), (5.10), transport equations for values $\left\langle\left\langle 1\left|\hat{p}_{n}\right| \varrho(t)\right\rangle\right\rangle$ and the corresponding equations for time correlation functions $\Phi_{n m}(t)$ in linear approximation (5.4) are closed.

If spectral magnitudes $\Phi_{n m}(\omega)$ for quantum time correlation functions $\Phi_{n m}(t)$ are defined as follows:

$$
\Phi_{n m}(\omega)=\frac{1}{2 \pi} \int_{-\infty}^{\infty} \mathrm{d} t \mathrm{e}^{\mathrm{i} \omega t} \Phi_{n m}(t),
$$

then, according to the definition of Green functions $[21,33]$ and the condition that $\Phi_{n m}(\omega)$ is a real function ( $\omega$ is a real number), one finds imaginary parts of spectral magnitudes of the corresponding retarded, advanced and causal Green functions

$$
\begin{aligned}
& \Im \mathfrak{m} G_{n m}^{\mathrm{r}, \mathrm{a}}(\omega)=\mp \frac{1}{2 \hbar}\left(\mathrm{e}^{\beta \hbar \omega}-\sigma\right) \Phi_{n m}(\omega), \\
& \Im \mathfrak{m} G_{n m}^{\mathrm{c}}(\omega)=-\frac{1}{2}\left(\mathrm{e}^{\beta \hbar \omega}+\sigma\right) \Phi_{n m}(\omega),
\end{aligned}
$$

where functions $G_{n m}^{\mathrm{r}}\left(t, t^{\prime}\right), G_{n m}^{\mathrm{a}}\left(t, t^{\prime}\right), G_{n m}^{\mathrm{c}}\left(t, t^{\prime}\right)$ in time representation constitute, correspondingly, retarded, advanced and causal Green functions in thermo field representation:

$$
\begin{aligned}
G_{n m}^{\mathrm{r}, \mathrm{a}}\left(t, t^{\prime}\right)= & \pm \frac{1}{\mathrm{i} \hbar} \theta\left(t-t^{\prime}\right)\left\langle\left\langle 1\left|\left[p_{n}(t), p_{m}\left(t^{\prime}\right)\right]_{\sigma}\right| \varrho_{0}\right\rangle\right\rangle= \\
& \pm \frac{1}{\mathrm{i} \hbar} \theta\left(t-t^{\prime}\right)\left\langle\left\langle 1\left|\left[\hat{p}_{n}(t), \hat{p}_{m}\left(t^{\prime}\right)\right]_{\sigma}\right| \varrho_{0}\right\rangle\right\rangle= \\
& \pm \frac{1}{\mathrm{i} \hbar} \theta\left(t-t^{\prime}\right)\left\langle\left\langle 1\left|\hat{p}_{n}(t) \hat{p}_{m}\left(t^{\prime}\right)-\sigma \hat{p}_{m}\left(t^{\prime}\right) \hat{p}_{n}(t)\right| \varrho_{0}\right\rangle\right\rangle \\
G_{n m}^{\mathrm{c}}\left(t, t^{\prime}\right)= & \frac{1}{\mathrm{i} \hbar}\left\langle\left\langle 1 \mid T p_{n}(t) p_{m}\left(t^{\prime}\right) \varrho_{0}\right\rangle\right\rangle \\
= & \frac{1}{\mathrm{i} \hbar} \theta\left(t-t^{\prime}\right)\left\langle\left\langle 1\left|\hat{p}_{n}(t) \hat{p}_{m}\left(t^{\prime}\right)\right| \varrho_{0}\right\rangle\right\rangle+\frac{1}{\mathrm{i} \hbar} \theta\left(t-t^{\prime}\right)\left\langle\left\langle 1\left|\tilde{p}_{n}^{+}(t) \hat{p}_{m}\left(t^{\prime}\right)\right| \varrho_{0}\right\rangle\right\rangle,
\end{aligned}
$$

where $\theta(t)=\left\{\begin{array}{l}1, t>0, \\ 0, t<0,\end{array}\right.$ is a unit step function. As it is seen from (5.15) and (5.16), retarded (advanced) and causal Green functions are defined via time correlation functions $\left\langle\left\langle 1\left|\hat{p}_{n}(t) \hat{p}_{m}\left(t^{\prime}\right)\right| \varrho_{0}\right\rangle\right\rangle,\left\langle\left\langle 1\left|\tilde{p}_{n}^{+}(t) \hat{p}_{m}\left(t^{\prime}\right)\right| \varrho_{0}\right\rangle\right\rangle$, which satisfy conditions (5.10). For completeness, let us write dispersion relations which connect the imaginary parts $\Im \mathfrak{m} G_{n m}^{\mathrm{r}, \mathrm{a}}(\omega), \Im \mathfrak{m} G_{n m}^{\mathrm{c}}(\omega)(5.13),(5.14)$ and the real parts $\Re \mathfrak{e} G_{n m}^{\mathrm{r}, \mathrm{a}}(\omega)$, $\Re \mathfrak{e} G_{n m}^{\mathrm{c}}(\omega)$ of spectral functions of the corresponding Green functions [21,33]:

$$
\begin{aligned}
& \Re \mathfrak{e} G_{n m}^{\mathrm{r}, \mathrm{a}}(\omega)= \pm \frac{1}{\pi} \mathscr{P} \int_{-\infty}^{\infty} \mathrm{d} \omega^{\prime} \frac{\Im \mathfrak{m} G^{\mathrm{r}, \mathrm{a}}\left(\omega^{\prime}\right)}{\omega^{\prime}-\omega} \\
& \Re \mathfrak{e} G_{n m}^{\mathrm{c}}(\omega)=\frac{1}{\pi} \mathscr{P} \int_{-\infty}^{\infty} \mathrm{d} \omega^{\prime} \frac{\Im \mathfrak{m} G^{\mathrm{c}}\left(\omega^{\prime}\right)}{\omega^{\prime}-\omega} \frac{\mathrm{e}^{\beta \hbar \omega^{\prime}}-\sigma}{\mathrm{e}^{\beta \hbar \omega^{\prime}}+\sigma} .
\end{aligned}
$$


It should be pointed out that Green functions in thermo field representation, which are calculated with the help of equilibrium thermo vacuum state vector $\left.\left|\varrho_{0}(\beta)\right\rangle\right\rangle$, were investigated in many papers $[32,38,40,41]$. Specifically, the diagram technique for their calculation, which generalizes the Feynman method, was developed in [42].

An application of the general structure of nonequilibrium thermo field dynamics on the basis of the nonequilibrium statistical operator method [21,27] will be considered in sections 7 and 8 . We will obtain equations of hydrodynamics and transport equations of a consistent description of the kinetics and hydrodynamics for dense quantum systems in thermo field representation. While investigating such systems, one of important problems is the calculation of transport cores (or transport coefficients) both for weakly and strongly nonequilibrium systems.

The problem is that quasiequilibrium thermo vacuum state vector $\left.\left|\varrho_{\mathrm{q}}(t)\right\rangle\right\rangle$, (3.10) or (5.4), in each case is not a vacuum state for superoperators $\hat{a}, \hat{a}^{+}, \tilde{a}$, $\tilde{a}^{+}$. The subject of this question consists in the construction of dynamical reflection of superoperators $\hat{a}, \hat{a}^{+}, \tilde{a}, \tilde{a}^{+}$into superoperators for "quasiparticles" for which the quasiequilibrium thermo vacuum state is a vacuum state. One method of constructing such superoperators is considered in the next section.

\section{Creation and annihilation superoperators of "quasiparticles" for the quasiequilibrium thermo vacuum state}

In our further consideration we assume that Hamiltonian $H(2.1)$ of a system can be represented in the form:

$$
H=H_{0}+H_{\text {int }},
$$

where $H_{\text {int }}$ contains a small parameter. This small parameter can be used for the construction of perturbation theory series. At the same time $H_{0}$ is a nonperturbed part of the Hamiltonian (6.1). It depends on creation and annihilation operators $a_{l}^{+}, a_{l}$ bilinearly. According to the nonequilibrium thermo field dynamics formalism, Hamiltonian $\bar{H}$ of a system reads:

$$
\begin{aligned}
\bar{H} & =\bar{H}_{0}+\bar{H}_{\text {int }}, \\
\bar{H}_{0} & =\hat{H}_{0}-\tilde{H}_{0}, \\
\bar{H}_{\text {int }} & =\hat{H}_{\text {int }}-\tilde{H}_{\text {int }} .
\end{aligned}
$$

$\bar{H}_{0}$ depends on superoperators $\hat{a}^{+}, \hat{a}, \tilde{a}^{+}, \tilde{a}$ bilinearly. In such a case, to construct the perturbation theory for operators it is convenient to use the Heisenberg representation on the non-perturbed part of Hamiltonian $\bar{H}_{0}$ :

$$
\bar{A}(t)=\exp \left\{-\frac{1}{\mathrm{i} \hbar} \bar{H}_{0} t\right\} \bar{A} \exp \left\{\frac{1}{\mathrm{i} \hbar} \bar{H}_{0} t\right\} .
$$

It is known that $\bar{A}(t)$ satisfies the Heisenberg equation

$$
\frac{\partial}{\partial t} \bar{A}(t)=-\frac{1}{\mathrm{i} \hbar}\left[\bar{H}_{\mathrm{int}}(t), \bar{A}(t)\right], \bar{H}_{\mathrm{int}}(t)=\exp \left\{-\frac{1}{\mathrm{i} \hbar} \bar{H}_{0} t\right\} \bar{H} \exp \left\{\frac{1}{\mathrm{i} \hbar} \bar{H}_{0} t\right\}
$$


Superoperators $\hat{a}^{+}, \hat{a}, \tilde{a}^{+}, \tilde{a}$ in the Heisenberg representation read:

$$
\begin{aligned}
& \hat{a}(t)=\mathrm{e}^{-\frac{1}{\mathrm{i} \hbar} \bar{H}_{0} t} \hat{a} \mathrm{e}^{\frac{1}{\mathrm{i} \hbar} \bar{H}_{0} t}, \quad \hat{a}^{+}(t)=\mathrm{e}^{-\frac{1}{\mathrm{i} \hbar} \bar{H}_{0} t} \hat{a}^{+} \mathrm{e}^{\frac{1}{\mathrm{i} \hbar} \bar{H}_{0} t}, \\
& \tilde{a}(t)=\mathrm{e}^{-\frac{1}{\mathrm{i} \hbar} \bar{H}_{0} t} \tilde{a} \mathrm{e}^{\frac{1}{i \hbar} \bar{H}_{0} t}, \quad \tilde{a}^{+}(t)=\mathrm{e}^{-\frac{1}{\mathrm{i} \hbar} \bar{H}_{0} t} \tilde{a}^{+} \mathrm{e}^{\frac{1}{\mathrm{i} \hbar} \bar{H}_{0} t} .
\end{aligned}
$$

They satisfy the "classical" commutation relations:

$$
\begin{array}{ll}
{\left[\hat{a}(t), \hat{a}^{+}(t)\right]_{\sigma}=\left[\tilde{a}(t), \tilde{a}^{+}(t)\right]_{\sigma}=1,} & {[\hat{a}(t), \tilde{a}(t)]_{\sigma}=\left[\hat{a}^{+}(t), \tilde{a}^{+}(t)\right]_{\sigma}=0,} \\
{[\hat{a}(t), \hat{a}(t)]_{\sigma}=\left[\hat{a}^{+}(t), \hat{a}^{+}(t)\right]_{\sigma}=0,} & {[\tilde{a}(t), \tilde{a}(t)]_{\sigma}=\left[\tilde{a}^{+}(t), \tilde{a}^{+}(t)\right]_{\sigma}=0 .}
\end{array}
$$

Let us assume that the quasiequilibrium thermo vacuum state vector describes the initial state of a quantum system by the non-perturbed part of Hamiltonian $\left.\bar{H}_{0} \cdot\left|\varrho_{\mathrm{q}}\left(t_{0}\right)\right\rangle\right\rangle$ is not a vacuum state for annihilation superoperators $\hat{a}(t), \tilde{a}(t)$, i.e.

$$
\begin{aligned}
\left.\hat{a}(t)\left|\varrho_{\mathrm{q}}\left(t_{0}\right)\right\rangle\right\rangle & \left.=f\left(t-t_{0}\right) \tilde{a}^{+}(t)\left|\varrho_{\mathrm{q}}\left(t_{0}\right)\right\rangle\right\rangle, \\
\left.\tilde{a}(t)\left|\varrho_{\mathrm{q}}\left(t_{0}\right)\right\rangle\right\rangle & \left.=\sigma f\left(t-t_{0}\right) \hat{a}^{+}(t)\left|\varrho_{\mathrm{q}}\left(t_{0}\right)\right\rangle\right\rangle, \\
\left\langle\langle 1| \hat{a}^{+}(t)\right. & =\sigma\left\langle\langle 1| \tilde{a}^{+}(t),\right.
\end{aligned}
$$

where function $f\left(t-t_{0}\right)$ will be defined below. Nevertheless, the linear combination of superoperators $\hat{a}^{+}, \hat{a}, \tilde{a}^{+}, \tilde{a}$ allows us to define new creation and annihilation superoperators $\hat{\gamma}^{+}, \hat{\gamma}(t)$ and $\tilde{\gamma}^{+}(t), \tilde{\gamma}(t)[35-37]$ :

$$
\begin{aligned}
\hat{\gamma}(t) & =Q^{1 / 2}\left(t-t_{0}\right)\left[\hat{a}(t)-f\left(t-t_{0}\right) \tilde{a}^{+}(t)\right], \\
\hat{\gamma}^{+}(t) & =Q^{1 / 2}\left(t-t_{0}\right)\left[\tilde{a}^{+}(t)-\sigma \hat{a}(t)\right] .
\end{aligned}
$$

So, taking into account (6.7), the action of new operators on state vectors reads:

$$
\begin{array}{ll}
\left.\hat{\gamma}(t)\left|\varrho_{\mathrm{q}}\left(t_{0}\right)\right\rangle\right\rangle=0, & \left\langle\langle 1| \hat{\gamma}^{+}(t)=0,\right. \\
\left.\tilde{\gamma}(t)\left|\varrho_{\mathrm{q}}\left(t_{0}\right)\right\rangle\right\rangle=0, & \left\langle\langle 1| \tilde{\gamma}^{+}(t)=0 .\right.
\end{array}
$$

Superoperators $\hat{\gamma}^{+}, \hat{\gamma}(t)$ and $\tilde{\gamma}^{+}(t), \tilde{\gamma}(t)$ satisfy the "canonical" commutation relations:

$$
\begin{aligned}
& {\left[\hat{\gamma}(t), \hat{\gamma}^{+}(t)\right]_{\sigma}=1,} \\
& {\left[\tilde{\gamma}(t), \tilde{\gamma}^{+}(t)\right]_{\sigma}=1, \quad[\hat{\gamma}(t), \tilde{\gamma}(t)]_{\sigma}=\left[\hat{\gamma}^{+}(t), \tilde{\gamma}^{+}(t)\right]_{\sigma}=0 .}
\end{aligned}
$$

A connecting expression between multiplier $Q\left(t-t_{0}\right)$ and function $f\left(t-t_{0}\right)$ may be found on the basis of relations (6.8), (6.9) and (6.11):

$$
Q\left(t-t_{0}\right)=\left[1-\sigma f\left(t-t_{0}\right)\right]^{-1} .
$$

But, to define $f\left(t-t_{0}\right)$ function let us use the second equality in (6.7). It aids to obtain the following:

$$
\left\langle\left\langle 1|\hat{a}(t) \tilde{a}(t)| \varrho_{\mathrm{q}}\left(t_{0}\right)\right\rangle\right\rangle=\sigma f\left(t-t_{0}\right)\left\langle\left\langle 1\left|\hat{a}(t) \hat{a}^{+}(t)\right| \varrho_{\mathrm{q}}\left(t_{0}\right)\right\rangle\right\rangle .
$$

And, using the third equality in (6.7) and (6.13) we arrive at

$$
\left\langle\left\langle 1\left|\tilde{a}^{+}(t) \tilde{a}(t)\right| \varrho_{\mathrm{q}}\left(t_{0}\right)\right\rangle\right\rangle=f\left(t-t_{0}\right)\left\langle\left\langle 1\left|\hat{a}(t) \hat{a}^{+}(t)\right| \varrho_{\mathrm{q}}\left(t_{0}\right)\right\rangle\right\rangle .
$$


As far as

$$
n\left(t-t_{0}\right)=\left\langle\left\langle 1\left|\tilde{a}^{+}(t) \tilde{a}(t)\right| \varrho_{\mathrm{q}}\left(t_{0}\right)\right\rangle\right\rangle=\left\langle\left\langle 1\left|\hat{a}^{+}(t) \hat{a}(t)\right| \varrho_{\mathrm{q}}\left(t_{0}\right)\right\rangle\right\rangle
$$

is the average particle number, then, using in the right-hand side of (6.14) commutation relation $\hat{a}(t) \hat{a}^{+}(t)-\sigma \hat{a}^{+}(t) \hat{a}(t)=1$, we obtain a linkage between $n\left(t-t_{0}\right)$ and function $f\left(t-t_{0}\right)$, and vice versa:

$$
\begin{aligned}
& n\left(t-t_{0}\right)=f\left(t-t_{0}\right)\left(1+\sigma n\left(t-t_{0}\right)\right), \\
& f\left(t-t_{0}\right)=n\left(t-t_{0}\right) /\left(1+\sigma n\left(t-t_{0}\right)\right) .
\end{aligned}
$$

Finally, if to substitute (6.16) into (6.12), one finds a linkage of normalized multiplier $Q\left(t-t_{0}\right)$ and average particle number $n\left(t-t_{0}\right)$ :

$$
Q\left(t-t_{0}\right)=1+\sigma n\left(t-t_{0}\right) .
$$

Now, taking into account (6.16) and (6.17), relations (6.8), (6.9) for superoperators $\hat{\gamma}^{+}, \hat{\gamma}(t)$, and $\tilde{\gamma}^{+}(t), \tilde{\gamma}(t)$ read:

$$
\begin{aligned}
\hat{\gamma}(t) & =\left(1+\sigma n\left(t-t_{0}\right)\right)^{\frac{1}{2}}\left[\hat{a}(t)-\frac{n\left(t-t_{0}\right)}{1+\sigma n\left(t-t_{0}\right)} \tilde{a}^{+}(t)\right], \\
\tilde{\gamma}^{+}(t) & =\left(1+\sigma n\left(t-t_{0}\right)\right)^{\frac{1}{2}}\left[\tilde{a}^{+}(t)-\sigma \hat{a}(t)\right] .
\end{aligned}
$$

Inversed transformations for superoperators $\hat{a}^{+}, \hat{a}(t)$ and $\tilde{a}^{+}(t), \tilde{a}(t)$ can be easily obtained from (6.18):

$$
\begin{aligned}
\hat{a}(t) & =\left(1+\sigma n\left(t-t_{0}\right)\right)^{\frac{1}{2}}\left[\hat{\gamma}(t)+\frac{n\left(t-t_{0}\right)}{1+\sigma n\left(t-t_{0}\right)} \tilde{\gamma}^{+}(t)\right], \\
\tilde{a}^{+}(t) & =\left(1+\sigma n\left(t-t_{0}\right)\right)^{\frac{1}{2}}\left[\tilde{\gamma}^{+}(t)+\sigma \hat{\gamma}(t)\right] .
\end{aligned}
$$

$\hat{\gamma}(t), \hat{\gamma}^{+}(t), \tilde{\gamma}(t), \tilde{\gamma}^{+}(t)$ can be defined as annihilation and creation superoperators of quasiparticles for which quasiequilibrium thermo vacuum state $\left.\left|\varrho_{\mathrm{q}}(t)\right\rangle\right\rangle$ is a vacuum state. These superoperators are functions of thermodynamic parameters $F_{n}(t)$ which describe the quasiequilibrium state of a system. Relations (6.18), (6.19) are a dynamical reflection between $\hat{a}(t), \hat{a}^{+}(t), \tilde{a}(t), \tilde{a}^{+}(t)$ and $\hat{\gamma}(t), \hat{\gamma}^{+}(t), \tilde{\gamma}(t)$, $\tilde{\gamma}^{+}(t)$. While calculating transport cores in transport equations (4.14) and (5.7), or connected with these quantities Green functions, superoperators $\hat{a}(t), \hat{a}^{+}(t)$, $\tilde{a}(t), \tilde{a}^{+}(t)$ are multiplied according to the Wick theorem about normal products $: \hat{a}^{+}(t) \hat{a}(t):,: \tilde{a}^{+}(t) \tilde{a}(t):$. Coming now to superoperators $\hat{\gamma}(t), \hat{\gamma}^{+}(t), \tilde{\gamma}(t), \tilde{\gamma}^{+}(t)$ with the help of inversed transformations (6.19), one can obtain for the calculation of transport cores or the corresponding Green functions, a generalization of the Wick formulae and Feynman diagram technique on a nonequilibrium case. It is one of important features of the nonequilibrium thermo field dynamics. It consists in the possibility of constructing the quasiequilibrium thermo vacuum state vector as a vacuum state vector for the solution to the Schrödinger equation (2.6) of a quantum field system. 
In the following section we consider equations of thermo field hydrodynamics of quantum systems of both strongly and weakly nonequilibrium states using the base transport equations (4.14). We obtain expressions for generalized transport coefficients of viscosity and thermal conductivity in thermo field representation.

\section{Generalized hydrodynamic equations in thermo field repre- sentation}

In the case of description of the nonequilibrium state of a quantum system the following quantities can be chosen as parameters of a shortened description: they are density operators of particle number $n_{\boldsymbol{k}}$, momentum $\boldsymbol{p}_{\boldsymbol{k}}$ and energy $E_{\boldsymbol{k}}$. For such a set of values quasiequilibrium statistical operator $\hat{\varrho}_{\mathrm{q}}(t)$ reads $[21,33,34]$ :

$$
\hat{\varrho_{\mathrm{q}}}(t)=\exp \left\{-\Phi(t)-\sum_{\boldsymbol{k}}\left[\beta_{-\boldsymbol{k}}(t) \hat{E}(t)-(\beta \boldsymbol{v})_{\boldsymbol{k}}(t) \hat{\boldsymbol{p}}_{\boldsymbol{k}}-\left[\beta\left(\mu-\frac{m}{2} V^{2}\right)\right]_{-\boldsymbol{k}}(t) \hat{n}_{\boldsymbol{k}}\right]\right\},
$$

where parameters $\mu_{-\boldsymbol{k}}(t), \boldsymbol{v}_{-\boldsymbol{k}}(t), \beta_{-\boldsymbol{k}}(t)$ are defined from the self-consistency conditions

$$
\begin{aligned}
& \left\langle\left\langle 1\left|\hat{n}_{\boldsymbol{k}}\right| \varrho_{\mathrm{q}}(t)\right\rangle\right\rangle=\left\langle\left\langle 1\left|\hat{n}_{\boldsymbol{k}}\right| \varrho(t)\right\rangle\right\rangle, \\
& \left\langle\left\langle 1\left|\hat{\boldsymbol{p}}_{\boldsymbol{k}}\right| \varrho_{\mathrm{q}}(t)\right\rangle\right\rangle=\left\langle\left\langle 1\left|\hat{\boldsymbol{p}}_{\boldsymbol{k}}\right| \varrho(t)\right\rangle\right\rangle, \\
& \left\langle\left\langle 1\left|\hat{E}_{\boldsymbol{k}}\right| \varrho_{\mathrm{q}}(t)\right\rangle\right\rangle=\left\langle\left\langle 1\left|\hat{E}_{\boldsymbol{k}}\right| \varrho(t)\right\rangle\right\rangle,
\end{aligned}
$$

and mean a chemical potential, average hydrodynamical velocity and a local value of inversed temperature, respectively [21]. Expression (7.1) aids to obtain a set of equations of generalized hydrodynamics in thermo field representation. Taking into account (4.14), we obtain:

$$
\begin{aligned}
& \frac{\partial}{\partial t}\left\langle\left\langle 1\left|\hat{n}_{\boldsymbol{k}}\right| \varrho(t)\right\rangle\right\rangle=\left\langle\left\langle 1\left|\dot{\hat{n}}_{\boldsymbol{k}}\right| \varrho_{\mathrm{q}}(t)\right\rangle\right\rangle, \\
& \frac{\partial}{\partial t}\left\langle\left\langle 1\left|\hat{\boldsymbol{p}}_{\boldsymbol{k}}\right| \varrho(t)\right\rangle\right\rangle=\left\langle\left\langle 1\left|\dot{\hat{\boldsymbol{p}}}_{\boldsymbol{k}}\right| \varrho_{\mathrm{q}}(t)\right\rangle\right\rangle- \\
& \sum_{\boldsymbol{g}} \int_{-\infty}^{t} \mathrm{~d} t^{\prime} \mathrm{e}^{\varepsilon\left(t^{\prime}-t\right)}\left\langle\left\langle J_{p}(\boldsymbol{k} ; t)\left|T\left(t, t^{\prime}\right)\right| \int_{0}^{1} \mathrm{~d} \tau \varrho_{\mathrm{q}}^{\tau}\left(t^{\prime}\right) J_{p}\left(\boldsymbol{g} ; t^{\prime}\right) \varrho_{\mathrm{q}}^{1-\tau}\left(t^{\prime}\right)\right\rangle\right\rangle(\beta \boldsymbol{v})_{\boldsymbol{g}}\left(t^{\prime}\right)+ \\
& \sum_{\boldsymbol{g}} \int_{-\infty}^{t} \mathrm{~d} t^{\prime} \mathrm{e}^{\varepsilon\left(t^{\prime}-t\right)}\left\langle\left\langle J_{p}(\boldsymbol{k} ; t)\left|T\left(t, t^{\prime}\right)\right| \int_{0}^{1} \mathrm{~d} \tau \varrho_{\mathrm{q}}^{\tau}\left(t^{\prime}\right) J_{E}\left(\boldsymbol{g} ; t^{\prime}\right) \varrho_{\mathrm{q}}^{1-\tau}\left(t^{\prime}\right)\right\rangle\right\rangle \beta_{\boldsymbol{g}}\left(t^{\prime}\right), \\
& \frac{\partial}{\partial t}\left\langle\left\langle 1\left|\hat{E}_{\boldsymbol{k}}\right| \varrho(t)\right\rangle\right\rangle=\left\langle\left\langle 1\left|\dot{\hat{E}}_{\boldsymbol{k}}\right| \varrho_{\mathrm{q}}(t)\right\rangle\right\rangle- \\
& \sum_{\boldsymbol{g}} \int_{-\infty}^{t} \mathrm{~d} t^{\prime} \mathrm{e}^{\varepsilon\left(t^{\prime}-t\right)}\left\langle\left\langle J_{E}(\boldsymbol{k} ; t)\left|T\left(t, t^{\prime}\right)\right| \int_{0}^{1} \mathrm{~d} \tau \varrho_{\mathrm{q}}^{\tau}\left(t^{\prime}\right) J_{p}\left(\boldsymbol{g} ; t^{\prime}\right) \varrho_{\mathrm{q}}^{1-\tau}\left(t^{\prime}\right)\right\rangle\right\rangle(\beta \boldsymbol{v})_{\boldsymbol{g}}\left(t^{\prime}\right)+
\end{aligned}
$$




$$
\sum_{\boldsymbol{g}} \int_{-\infty}^{t} \mathrm{~d} t^{\prime} \mathrm{e}^{\varepsilon\left(t^{\prime}-t\right)}\left\langle\left\langle J_{E}(\boldsymbol{k} ; t)\left|T\left(t, t^{\prime}\right)\right| \int_{0}^{1} \mathrm{~d} \tau \varrho_{\mathrm{q}}^{\tau}\left(t^{\prime}\right) J_{E}\left(\mathbf{g} ; t^{\prime}\right) \varrho_{\mathrm{q}}^{1-\tau}\left(t^{\prime}\right)\right\rangle\right\rangle \beta_{\boldsymbol{g}}\left(t^{\prime}\right)
$$

where

$$
\begin{array}{ll}
\hat{n}_{\boldsymbol{k}}=n_{\boldsymbol{k}}\left(\hat{a}_{\boldsymbol{k}}^{+}, \hat{a}_{\boldsymbol{k}}\right), & \\
\hat{\boldsymbol{p}}_{\boldsymbol{k}}=\boldsymbol{p}_{\boldsymbol{k}}\left(\hat{a}_{\boldsymbol{k}}^{+}, \hat{a}_{\boldsymbol{k}}\right), & J_{p}(\boldsymbol{k} ; t)=(1-\mathscr{P}(t)) \dot{\boldsymbol{p}}_{\boldsymbol{k}}, \\
\hat{E}_{\boldsymbol{k}}=E_{\boldsymbol{k}}\left(\hat{a}_{\boldsymbol{k}}^{+}, \hat{a}_{\boldsymbol{k}}\right), & J_{E}(\boldsymbol{k} ; t)=(1-\mathscr{P}(t)) \dot{E}_{\boldsymbol{k}},
\end{array}
$$

are generalized flows. But the Mori projection operator $\mathscr{P}(t)$ at the description of the hydrodynamic state reads:

$$
\mathscr{P}(t)(\ldots)=\left\langle\left\langle 1 \mid \ldots \varrho_{\mathrm{q}}(t)\right\rangle\right\rangle+\sum_{a=\{n, \boldsymbol{p}, E\}} \sum_{\boldsymbol{k}}\left[\frac{\delta\left\langle\langle 1| \ldots \varrho_{\mathrm{q}}(t)\right.}{\delta\left\langle\left\langle 1\left|\hat{a}_{\boldsymbol{k}}\right| \varrho(t)\right\rangle\right\rangle}\left(a_{\boldsymbol{k}}-\left\langle\left\langle 1\left|\hat{a}_{\boldsymbol{k}}\right| \varrho(t)\right\rangle\right\rangle\right)\right] .
$$

Transport cores

$$
\begin{aligned}
& \left\langle\left\langle J_{p}(\boldsymbol{k} ; t)\left|T\left(t, t^{\prime}\right)\right| \int_{0}^{1} \mathrm{~d} \tau \varrho_{\mathrm{q}}^{\tau}\left(t^{\prime}\right) J_{p}\left(\mathbf{g} ; t^{\prime}\right) \varrho_{\mathrm{q}}^{1-\tau}\left(t^{\prime}\right)\right\rangle\right\rangle, \\
& \left\langle\left\langle J_{p}(\boldsymbol{k} ; t)\left|T\left(t, t^{\prime}\right)\right| \int_{0}^{1} \mathrm{~d} \tau \varrho_{\mathrm{q}}^{\tau}\left(t^{\prime}\right) J_{E}\left(\mathbf{g} ; t^{\prime}\right) \varrho_{\mathrm{q}}^{1-\tau}\left(t^{\prime}\right)\right\rangle\right\rangle, \\
& \left\langle\left\langle J_{E}(\boldsymbol{k} ; t)\left|T\left(t, t^{\prime}\right)\right| \int_{0}^{1} \mathrm{~d} \tau \varrho_{\mathrm{q}}^{\tau}\left(t^{\prime}\right) J_{p}\left(\mathbf{g} ; t^{\prime}\right) \varrho_{\mathrm{q}}^{1-\tau}\left(t^{\prime}\right)\right\rangle\right\rangle, \\
& \left\langle\left\langle J_{E}(\boldsymbol{k} ; t)\left|T\left(t, t^{\prime}\right)\right| \int_{0}^{1} \mathrm{~d} \tau \varrho_{\mathrm{q}}^{\tau}\left(t^{\prime}\right) J_{E}\left(\mathbf{g} ; t^{\prime}\right) \varrho_{\mathrm{q}}^{1-\tau}\left(t^{\prime}\right)\right\rangle\right\rangle,
\end{aligned}
$$

in (7.4), (7.5) are calculated with the help of quasiequilibrium thermo vacuum state vector (7.1).

For the description of nonequilibrium hydrodynamic state of a quantum field system near equilibrium, the set of equations of generalized hydrodynamics (7.3)(7.5) according to (5.1)-(5.5) becomes closed:

$$
\begin{aligned}
& \frac{\partial}{\partial t}\left\langle\left\langle 1\left|\delta \hat{n}_{\boldsymbol{k}}\right| \varrho(t)\right\rangle\right\rangle=\sum_{\boldsymbol{g}} \Omega_{n \boldsymbol{p}}(\boldsymbol{k}, \mathbf{g})\left\langle\left\langle 1\left|\delta \hat{\boldsymbol{p}}_{\boldsymbol{g}}\right| \varrho(t)\right\rangle\right\rangle, \\
& \frac{\partial}{\partial t}\left\langle\left\langle 1\left|\delta \hat{\boldsymbol{p}}_{\boldsymbol{k}}\right| \varrho(t)\right\rangle\right\rangle=\sum_{\boldsymbol{g}} \Omega_{\boldsymbol{p} n}(\boldsymbol{k}, \mathbf{g})\left\langle\left\langle 1\left|\delta \hat{n}_{\boldsymbol{g}}\right| \varrho(t)\right\rangle\right\rangle+ \\
& \sum_{\boldsymbol{g}} \Omega_{\boldsymbol{p} h}(\boldsymbol{k}, \boldsymbol{g})\left\langle\left\langle 1\left|\delta \hat{h}_{\boldsymbol{g}}\right| \varrho(t)\right\rangle\right\rangle- \\
& \sum_{\boldsymbol{g}} \int_{-\infty}^{t} \mathrm{~d} t^{\prime} \mathrm{e}^{\varepsilon\left(t^{\prime}-t\right)} \boldsymbol{k}: \eta\left(\boldsymbol{k}, \boldsymbol{g} ; t, t^{\prime}\right): \mathbf{g}\left\langle\left\langle 1\left|\delta \hat{\boldsymbol{p}}_{\boldsymbol{g}}\right| \varrho\left(t^{\prime}\right)\right\rangle\right\rangle-
\end{aligned}
$$




$$
\begin{gathered}
\sum_{\boldsymbol{g}} \int_{-\infty}^{t} \mathrm{~d} t^{\prime} \mathrm{e}^{\varepsilon\left(t^{\prime}-t\right)} \boldsymbol{k}: \xi_{\boldsymbol{p} h}\left(\boldsymbol{k}, \mathbf{g} ; t, t^{\prime}\right) \cdot \mathbf{g}\left\langle\left\langle 1\left|\delta \hat{h}_{\boldsymbol{g}}\right| \varrho\left(t^{\prime}\right)\right\rangle\right\rangle, \\
\frac{\partial}{\partial t}\left\langle\left\langle 1\left|\delta \hat{h}_{\boldsymbol{k}}\right| \varrho(t)\right\rangle\right\rangle=\sum_{\boldsymbol{g}} \Omega_{h \boldsymbol{p}}(\boldsymbol{k}, \mathbf{g})\left\langle\left\langle 1\left|\delta \hat{\boldsymbol{p}}_{\boldsymbol{g}}\right| \varrho(t)\right\rangle\right\rangle- \\
\sum_{\boldsymbol{g}} \int_{-\infty}^{t} \mathrm{~d} t^{\prime} \mathrm{e}^{\varepsilon\left(t^{\prime}-t\right)} \boldsymbol{k}: \xi_{h \boldsymbol{p}}\left(\boldsymbol{k}, \mathbf{g} ; t, t^{\prime}\right): \mathbf{g}\left\langle\left\langle 1\left|\delta \hat{\boldsymbol{p}}_{\boldsymbol{g}}\right| \varrho\left(t^{\prime}\right)\right\rangle\right\rangle- \\
\sum_{\boldsymbol{g}} \int_{-\infty}^{t} \mathrm{~d} t^{\prime} \mathrm{e}^{\varepsilon\left(t^{\prime}-t\right)} \boldsymbol{k} \cdot \lambda\left(\boldsymbol{k}, \mathbf{g} ; t, t^{\prime}\right) \cdot \mathbf{g}\left\langle\left\langle 1\left|\delta \hat{h}_{\boldsymbol{g}}\right| \varrho\left(t^{\prime}\right)\right\rangle\right\rangle .
\end{gathered}
$$

In these relations all quantities $\Omega_{n \boldsymbol{p}}(\boldsymbol{k}, \mathbf{g}), \Omega_{\boldsymbol{p} n}(\boldsymbol{k}, \mathbf{g}), \Omega_{\boldsymbol{p h}}(\boldsymbol{k}, \mathbf{g})$ and $\Omega_{h \boldsymbol{p}}(\boldsymbol{k}, \mathbf{g})$ are defined by the relation (5.6) and are equilibrium quantum correlation functions; $\eta\left(\boldsymbol{k}, \mathbf{g} ; t, t^{\prime}\right), \lambda\left(\boldsymbol{k}, \mathbf{g} ; t, t^{\prime}\right), \xi_{\boldsymbol{p} h}\left(\boldsymbol{k}, \mathbf{g} ; t, t^{\prime}\right), \xi_{h \boldsymbol{p}}\left(\boldsymbol{k}, \mathbf{g} ; t, t^{\prime}\right)$ are, correspondingly, time-dependent generalized transport coefficients of viscosity, thermal conductivity and cross transport coefficients. They are defined as follows:

$$
\begin{aligned}
& \boldsymbol{k}: \eta\left(\boldsymbol{k}, \mathbf{g} ; t, t^{\prime}\right): \mathbf{g}=\left\langle\left\langle J_{\boldsymbol{p}}(\boldsymbol{k}) T_{0}\left(t, t^{\prime}\right) \mid \int_{0}^{1} \mathrm{~d} \tau \varrho_{0}^{\tau} J_{\boldsymbol{p}}(\mathbf{g}) \varrho_{0}^{1-\tau}\right\rangle\right\rangle \chi_{\boldsymbol{p} \boldsymbol{p}}^{-1}(\mathbf{g}), \\
& \boldsymbol{k}: \xi_{\boldsymbol{p} h}\left(\boldsymbol{k}, \mathbf{g} ; t, t^{\prime}\right) \cdot \boldsymbol{k}=\left\langle\left\langle J_{\boldsymbol{p}}(\boldsymbol{k}) T_{0}\left(t, t^{\prime}\right) \mid \int_{0}^{1} \mathrm{~d} \tau \varrho_{0}^{\tau} J_{h}(\mathbf{g}) \varrho_{0}^{1-\tau}\right\rangle\right\rangle \chi_{h h}^{-1}(\mathbf{g}), \\
& \boldsymbol{k} \cdot \xi_{h \boldsymbol{p}}\left(\boldsymbol{k}, \mathbf{g} ; t, t^{\prime}\right): \mathbf{g}=\left\langle\left\langle J_{h}(\boldsymbol{k}) T_{0}\left(t, t^{\prime}\right) \mid \int_{0}^{1} \mathrm{~d} \tau \varrho_{0}^{\tau} J_{\boldsymbol{p}}(\mathbf{g}) \varrho_{0}^{1-\tau}\right\rangle\right\rangle \chi_{\boldsymbol{p} \boldsymbol{p}}^{-1}(\mathbf{g}), \\
& \boldsymbol{k} \cdot \lambda\left(\boldsymbol{k}, \mathbf{g} ; t, t^{\prime}\right) \cdot \mathbf{g}=\left\langle\left\langle J_{h}(\boldsymbol{k}) T_{0}\left(t, t^{\prime}\right) \mid \int_{0}^{1} \mathrm{~d} \tau \varrho_{0}^{\tau} J_{h}(\mathbf{g}) \varrho_{0}^{1-\tau}\right\rangle\right\rangle \chi_{h h}^{-1}(\mathbf{g}),
\end{aligned}
$$

where

$$
\begin{aligned}
& J_{p}(\boldsymbol{k})=\left(1-\mathscr{P}_{0}\right) \dot{\boldsymbol{p}}_{\boldsymbol{k}}, \\
& J_{h}(\boldsymbol{k})=\left(1-\mathscr{P}_{0}\right) \dot{h}_{\boldsymbol{k}}, \quad h_{\boldsymbol{k}}=E_{\boldsymbol{k}}-\chi_{E n}(\boldsymbol{k}) \chi_{n n}^{-1}(\boldsymbol{k}) n_{\boldsymbol{k}},
\end{aligned}
$$

and $\chi_{p p}(\boldsymbol{k}), \chi_{h h}(\boldsymbol{k}), \chi_{E n}(\boldsymbol{k}), \chi_{n n}(\boldsymbol{k})$ are equilibrium quantum correlation functions:

$$
\begin{array}{ll}
\chi_{\boldsymbol{p} \boldsymbol{p}}(\mathbf{g})=\left\langle\left\langle\boldsymbol{p}_{\boldsymbol{g}} \mid \int_{0}^{1} \mathrm{~d} \tau \varrho_{0}^{\tau} \boldsymbol{p}_{-\mathbf{g}} \varrho_{0}^{1-\tau}\right\rangle\right\rangle, & \chi_{h h}(\mathbf{g})=\left\langle\left\langle h_{\boldsymbol{g}} \mid \int_{0}^{1} \mathrm{~d} \tau \varrho_{0}^{\tau} h_{-\mathbf{g}} \varrho_{0}^{1-\tau}\right\rangle\right\rangle, \\
\chi_{n n}(\mathbf{g})=\left\langle\left\langle n_{\boldsymbol{g}} \mid \int_{0}^{1} \mathrm{~d} \tau \varrho_{0}^{\tau} n_{-\mathbf{g}} \varrho_{0}^{1-\tau}\right\rangle\right\rangle, & \chi_{E n}(\mathbf{g})=\left\langle\left\langle E_{\boldsymbol{g}} \mid \int_{0}^{1} \mathrm{~d} \tau \varrho_{0}^{\tau} n_{-\mathbf{g}} \varrho_{0}^{1-\tau}\right\rangle\right\rangle .
\end{array}
$$

In (7.15) the Mori projection operator $\mathscr{P}_{0}$ is defined in accordance with (5.8) and is built on operators $n_{\boldsymbol{k}}, \boldsymbol{p}_{\boldsymbol{k}}, h_{\boldsymbol{k}}$ in thermo field representation. Operator $h_{\boldsymbol{k}}$ appears due to the inclusion into $\left.\left|\varrho_{\mathrm{q}}(t)\right\rangle\right\rangle$ (7.1) parameters $\beta_{\boldsymbol{k}}(t)$ and $\mu_{\boldsymbol{k}}(t)$ with the help of self-consistency conditions (7.2) in the linear approximation (see (5.1)-(5.4)) 
and has the meaning of generalized enthalpy. On the basis of hydrodynamic equations (7.8)-(7.10) one can investigate quantum correlation functions like "densitydensity" $\Phi_{n n}(\boldsymbol{k} ; t)$, "flow-flow" $\Phi_{\boldsymbol{p} \boldsymbol{p}}(\boldsymbol{k} ; t)$, "enthalpy-enthalpy" $\Phi_{h h}(\boldsymbol{k} ; t)$ and the corresponding Green functions for specific quantum field systems.

A separate and very important problem is the calculation of transport coefficients (7.11)-(7.14). Consideration of nonequilibrium thermodynamics in a field theory on the basis of nonequilibrium statistical operator [33] and approximate calculation of viscosity and thermal conductivity coefficients for the $\Phi^{4}$ field model were done in paper [43]. These calculations were carried out using the Green-Kubo formulae which connect transport coefficients of viscosity and thermal conductivity with the corresponding Green functions. The last ones are built on the stress tensor and energy flow operators. In their turn, the Green functions were calculated using the Dzyaloshinski diagram method [44].

\section{Transport equations of a consistent description of the kinetics and hydrodynamics of dense quantum systems}

In the studies of nonequilibrium states of quantum field systems, such as a nuclear matter $[8,9]$, there arises a problem of taking into consideration coupled states. Kinetic and hydrodynamic processes in a hot, compressed nuclear matter, which appears after ultrarelativistic collisions of heavy nuclei or laser thermonuclear synthesis, are mutually connected and we should consider coupled states between nuclons. This is of great importance for the analysis and correlation of final reaction products. Obviously, a nuclon interaction investigation based on a quark-gluon plasma is a sequential microscopic approach to the dynamical description of reactions in a nuclear matter. The problem of a consistent description of the kinetics and hydrodynamics of a dense quark-gluon plasma is considered in section 9. For the description of kinetic processes in a nuclear matter on the level of model interactions, the Vlasov-Ueling-Uhlenbeck kinetic equation is used. This equation is used mainly in the case of low densities. The problems of a dense quark-gluon matter were discussed in detail in [8,9,20,45-47].

We will consider a quantum field system in which coupled states can appear between the particles. Let us introduce annihilation and creation operators of a coupled state $(A \alpha)$ with $A$-particle:

$$
\begin{aligned}
& a_{A \alpha}(\boldsymbol{p})=\sum_{1, \ldots, A} \Psi_{A \alpha \boldsymbol{p}}(1, \ldots, A) a(1) \ldots a(A), \\
& a_{A \alpha}^{+}(\boldsymbol{p})=\sum_{1, \ldots, A} \Psi_{A \alpha \boldsymbol{p}}^{*}(1, \ldots, A) a^{+}(1) \ldots a^{+}(A),
\end{aligned}
$$

where $\Psi_{A \alpha p}(1, \ldots, A)$ is a self-function of the $A$-particle coupled state, $\alpha$ denotes internal quantum numbers (spin, etc.), $\boldsymbol{p}$ is a particle momentum, the sum covers the particles. Annihilation and creation operators $a(j)$ and $a^{+}(j)$ satisfy the following commutation relations:

$$
\left[a(l), a^{+}(j)\right]_{\sigma}=\delta_{l, j}, \quad[a(l), a(j)]_{\sigma}=\left[a^{+}(l), a^{+}(j)\right]_{\sigma}=0,
$$


where $\sigma$-commutator is determined by $[a, b]_{\sigma}=a b-\sigma b a$ with $\sigma= \pm 1:+1$ for bosons and -1 for fermions.

The Hamiltonian of such a system can be written in the form:

$$
\begin{aligned}
& H=\sum_{A, \alpha} \int \frac{\mathrm{d} \boldsymbol{p} \mathrm{d} \boldsymbol{q}}{(2 \pi \hbar)^{6}} \frac{p^{2}}{2 m_{A}} a_{A \alpha}^{+}\left(\boldsymbol{p}-\frac{\boldsymbol{q}}{2}\right) a_{A \alpha}\left(\boldsymbol{p}+\frac{\boldsymbol{q}}{2}\right)+ \\
& \frac{1}{2} \sum_{A, B} \sum_{\alpha, \beta} \int \frac{\mathrm{d} \boldsymbol{p} \mathrm{d} \boldsymbol{p}^{\prime} \mathrm{d} \boldsymbol{q}}{(2 \pi \hbar)^{9}} V_{A B}(\boldsymbol{q}) a_{A \alpha}^{+}\left(\boldsymbol{p}+\frac{\boldsymbol{q}-\boldsymbol{p}^{\prime}}{2}\right) \hat{n}_{B \beta}(\boldsymbol{q}) a_{A \alpha}\left(\boldsymbol{p}-\frac{\boldsymbol{q}-\boldsymbol{p}^{\prime}}{2}\right),
\end{aligned}
$$

where $V_{A B}(\boldsymbol{q})$ is interaction energy between $A$ - and $B$-particle coupled states, $\boldsymbol{q}$ is a wavevector. Annihilation and creation operators $a_{A \alpha}(\boldsymbol{p})$ and $a_{A \alpha}^{+}(\boldsymbol{p})$ satisfy the following commutation relations:

$$
\begin{aligned}
& {\left[a_{A \alpha}(\boldsymbol{p}), a_{B \beta}^{+}\left(\boldsymbol{p}^{\prime}\right)\right]_{\sigma}=\delta_{A, B} \delta_{\alpha, \beta} \delta\left(\boldsymbol{p}-\boldsymbol{p}^{\prime}\right)} \\
& {\left[a_{A \alpha}(\boldsymbol{p}), a_{B \beta}\left(\boldsymbol{p}^{\prime}\right)\right]_{\sigma}=\left[a_{A \alpha}^{+}(\boldsymbol{p}), a_{B \beta}^{+}\left(\boldsymbol{p}^{\prime}\right)\right]_{\sigma}=0 .}
\end{aligned}
$$

$\hat{n}_{B \beta}(\boldsymbol{q})$ in $(8.3)$ is a Fourier transform of the $B$-particle density operator:

$$
\hat{n}_{B \beta}(\boldsymbol{q})=\int \frac{\mathrm{d} \boldsymbol{p}}{(2 \pi \hbar)^{3}} a_{\boldsymbol{p}-\frac{\boldsymbol{q}}{2}}^{+} a_{\boldsymbol{p}+\frac{\boldsymbol{q}}{2}} .
$$

As parameters of a shortened description for the consistent description of the kinetics and hydrodynamics of a system, where coupled states between the particles can appear, let us choose nonequilibrium distribution functions of $A$-particle coupled states in thermo field representation

$$
\left\langle\left\langle 1\left|\hat{n}_{A \alpha}(\boldsymbol{r}, \boldsymbol{p})\right| \varrho(t)\right\rangle\right\rangle=f_{A \alpha}(\boldsymbol{r}, \boldsymbol{p} ; t)=f_{A \alpha}(x ; t), \quad x=\{\boldsymbol{r}, \boldsymbol{p}\},
$$

here $f_{A \alpha}(x ; t)$ is a Wigner function of the $A$-particle coupled state where

$$
\hat{n}_{A \alpha}(\boldsymbol{r}, \boldsymbol{p}) \equiv \hat{n}_{A \alpha}(x)=\int \frac{\mathrm{d} \boldsymbol{q}}{(2 \pi \hbar)^{3}} \mathrm{e}^{-\frac{1}{\mathrm{i} \hbar} \boldsymbol{q} \cdot \boldsymbol{r}} \hat{a}_{A \alpha}^{+}\left(\boldsymbol{p}-\frac{\boldsymbol{q}}{2}\right) \hat{a}_{A \alpha}\left(\boldsymbol{p}+\frac{\boldsymbol{q}}{2}\right)
$$

is the Klimontovich density operator; and the average value of the total energy density operator

$$
\langle\langle 1|\hat{H}(\boldsymbol{r})| \varrho(t)\rangle\rangle=\langle\langle 1 \mid H(\boldsymbol{r}) \varrho(t)\rangle\rangle .
$$

By this $\int \mathrm{d} \boldsymbol{r} H(\boldsymbol{r})=H, \hat{H}(\boldsymbol{r})$ is a superoperator of the total energy density which is constructed on annihilation and creation superoperators $\hat{a}_{A \alpha}(\boldsymbol{p})$ and $\hat{a}_{A \alpha}^{+}(\boldsymbol{p})$. The latter satisfy commutation relations (8.4). Following [27], one can rewrite quasiequilibrium statistical operator $\left.\left.\hat{\varrho}_{\mathrm{q}}(t),\left|\varrho_{\mathrm{q}}(t)\right\rangle\right\rangle=\hat{\varrho}_{\mathrm{q}}(t)|1\rangle\right\rangle$ for the mentioned parameters of a shortened description in the form:

$$
\hat{\varrho}_{\mathrm{q}}(t)=\exp \left\{-\Phi^{*}(t)-\int \mathrm{d} \boldsymbol{r} \beta(\boldsymbol{r} ; t)\left(\hat{H}(\boldsymbol{r})-\sum_{A, \alpha} \int \frac{\mathrm{d} \boldsymbol{p}}{(2 \pi \hbar)^{3}} \mu_{A \alpha}(x ; t) \hat{n}_{A \alpha}(x)\right)\right\},
$$


where Lagrange multipliers $\beta(\boldsymbol{r} ; t)$ and $\mu_{A \alpha}(x ; t)$ can be found from the self-consistency conditions, correspondingly:

$$
\begin{aligned}
\langle\langle 1|\hat{H}(\boldsymbol{r})| \varrho(t)\rangle\rangle & =\left\langle\left\langle 1|\hat{H}(\boldsymbol{r})| \varrho_{\mathrm{q}}(t)\right\rangle\right\rangle, \\
\left\langle\left\langle 1\left|\hat{n}_{A \alpha}(x)\right| \varrho(t)\right\rangle\right\rangle & =\left\langle\left\langle 1\left|\hat{n}_{A \alpha}(x)\right| \varrho_{\mathrm{q}}(t)\right\rangle\right\rangle,
\end{aligned}
$$

$\Phi^{*}(t)$ is the Massieu-Planck functional and it can be defined from the normalization condition (3.6):

$$
\Phi^{*}(t)=\ln \left\langle\left\langle 1 \mid \exp \left\{-\int \mathrm{d} \boldsymbol{r} \beta(\boldsymbol{r} ; t)\left(\hat{H}(\boldsymbol{r})-\sum_{A, \alpha} \int \frac{\mathrm{d} \boldsymbol{p}}{(2 \pi \hbar)^{3}} \mu_{A \alpha}(x ; t) \hat{n}_{A \alpha}(x)\right)\right\}\right\rangle .\right.
$$

Using now the general structure of nonequilibrium thermo field dynamics (4.1)(4.14), one can obtain a set of generalized transport equations for $A$-particle Wigner distribution functions and the average interaction energy:

$$
\begin{aligned}
& \frac{\partial}{\partial t}\left\langle\left\langle 1\left|\hat{n}_{A \alpha}(x)\right| \varrho(t)\right\rangle\right\rangle=\left\langle\left\langle 1\left|\dot{\hat{n}}_{A \alpha}(x)\right| \varrho_{\mathrm{q}}(t)\right\rangle\right\rangle+ \\
& \int \mathrm{d} \boldsymbol{r}^{\prime} \int_{-\infty}^{t} \mathrm{~d} t^{\prime} \mathrm{e}^{\varepsilon\left(t^{\prime}-t\right)} \varphi_{n H}^{A \alpha}\left(x, \boldsymbol{r}^{\prime} ; t, t^{\prime}\right) \beta\left(\boldsymbol{r}^{\prime} ; t^{\prime}\right)+ \\
& \sum_{B, \beta} \int \mathrm{d} x^{\prime} \int_{-\infty}^{t} \mathrm{~d} t^{\prime} \mathrm{e}^{\varepsilon\left(t^{\prime}-t\right)} \varphi_{n n}^{A \alpha B \beta}\left(x, x^{\prime} ; t, t^{\prime}\right) \beta\left(\boldsymbol{r}^{\prime} ; t^{\prime}\right) \mu_{B \beta}\left(x^{\prime} ; t^{\prime}\right), \\
& \frac{\partial}{\partial t}\langle\langle 1|\hat{H}(\boldsymbol{r})| \varrho(t)\rangle\rangle=\left\langle\left\langle 1|\dot{\hat{H}}(\boldsymbol{r})| \varrho_{\mathrm{q}}(t)\right\rangle\right\rangle+ \\
& \int \mathrm{d} \boldsymbol{r}^{\prime} \int_{-\infty}^{t} \mathrm{~d} t^{\prime} \mathrm{e}^{\varepsilon\left(t^{\prime}-t\right)} \varphi_{H H}\left(\boldsymbol{r}, \boldsymbol{r}^{\prime} ; t, t^{\prime}\right) \beta\left(\boldsymbol{r}^{\prime} ; t^{\prime}\right)+ \\
& \sum_{B, \beta} \int_{-\infty}^{t} \mathrm{~d} x^{\prime} \int_{-\infty}^{t} \mathrm{~d} t^{\prime} \mathrm{e}^{\varepsilon\left(t^{\prime}-t\right)} \varphi_{H n}^{B \beta}\left(\boldsymbol{r}, x^{\prime} ; t, t^{\prime}\right) \beta\left(\boldsymbol{r}^{\prime} ; t^{\prime}\right) \mu_{B \beta}\left(x^{\prime} ; t^{\prime}\right),
\end{aligned}
$$

where $x^{\prime}=\left\{\boldsymbol{r}^{\prime}, \boldsymbol{p}^{\prime}\right\}, d x^{\prime}=(2 \pi \hbar)^{-3} d \boldsymbol{r}^{\prime} d \boldsymbol{p}^{\prime}$. Here

$$
\begin{aligned}
\varphi_{n n}^{A \alpha}\left(x, x^{\prime} ; t, t^{\prime}\right) & =\left\langle\left\langle 1\left|\hat{J}_{n_{A \alpha}}(x, t) T\left(t, t^{\prime}\right)\right| \int_{0}^{1} \mathrm{~d} \tau \varrho_{\mathrm{q}}^{\tau}\left(t^{\prime}\right) J_{n_{B \beta}}\left(x^{\prime} ; t^{\prime}\right) \varrho_{\mathrm{q}}^{1-\tau}\left(t^{\prime}\right)\right\rangle\right\rangle,(8 \\
\varphi_{n H}^{A \alpha}\left(x, \boldsymbol{r}^{\prime} ; t, t^{\prime}\right) & =\left\langle\left\langle 1\left|\hat{J}_{n_{A \alpha}}(x, t) T\left(t, t^{\prime}\right)\right| \int_{0}^{1} \mathrm{~d} \tau \varrho_{\mathrm{q}}^{\tau}\left(t^{\prime}\right) J_{H}\left(\boldsymbol{r}^{\prime} ; t^{\prime}\right) \varrho_{\mathrm{q}}^{1-\tau}\left(t^{\prime}\right)\right\rangle\right\rangle,( \\
\varphi_{H n}^{B \beta}\left(\boldsymbol{r}^{\prime}, x^{\prime} ; t, t^{\prime}\right) & =\left\langle\left\langle 1\left|\hat{J}_{H}(\boldsymbol{r}, t) T\left(t, t^{\prime}\right)\right| \int_{0}^{1} \mathrm{~d} \tau \varrho_{\mathrm{q}}^{\tau}\left(t^{\prime}\right) J_{n_{B \beta}}\left(x^{\prime} ; t^{\prime}\right) \varrho_{\mathrm{q}}^{1-\tau}\left(t^{\prime}\right)\right\rangle\right\rangle,( \\
\varphi_{H H}\left(\boldsymbol{r}, \boldsymbol{r}^{\prime} ; t, t^{\prime}\right) & =\left\langle\left\langle 1\left|\hat{J}_{H}(\boldsymbol{r}, t) T\left(t, t^{\prime}\right)\right| \int_{0}^{1} \mathrm{~d} \tau \varrho_{\mathrm{q}}^{\tau}\left(t^{\prime}\right) J_{H}\left(\boldsymbol{r}^{\prime} ; t^{\prime}\right) \varrho_{\mathrm{q}}^{1-\tau}\left(t^{\prime}\right)\right\rangle\right\rangle,
\end{aligned}
$$


are generalized transport cores which describe dissipative processes. In these formulae

$$
\begin{aligned}
& J_{H}(\boldsymbol{r} ; t)=\left(1-\mathscr{P}\left(t^{\prime}\right)\right) \dot{H}(\boldsymbol{r}), \\
& J_{n_{A \alpha}}(\boldsymbol{r}, \boldsymbol{p} ; t)=\left(1-\mathscr{P}\left(t^{\prime}\right)\right) \dot{n}_{A \alpha}(x)
\end{aligned}
$$

are generalized flows,

$$
\begin{aligned}
& \dot{H}(\boldsymbol{r})=-\frac{1}{\mathrm{i} \hbar}[H, H(\boldsymbol{r})], \\
& \dot{n}_{A \alpha}(\boldsymbol{r}, \boldsymbol{p})=-\frac{1}{\mathrm{i} \hbar}\left[H, n_{A \alpha}(x)\right],
\end{aligned}
$$

$\mathscr{P}(t)$ is a generalized Mori projection operator in thermo field representation. It acts on operators

$$
\begin{aligned}
& \mathscr{P}(t) P=\left\langle\left\langle|\hat{P}| \varrho_{\mathrm{q}}(t)\right\rangle\right\rangle+ \\
& \int \mathrm{d} \boldsymbol{r} \frac{\delta\left\langle\left\langle 1|\hat{P}| \varrho_{\mathrm{q}}(t)\right\rangle\right\rangle}{\delta\langle\langle 1|\hat{H}(\boldsymbol{r})| \varrho(t)\rangle\rangle}(H(\boldsymbol{r})-\langle\langle 1|\hat{H}(\boldsymbol{r})| \varrho(t)\rangle\rangle)+ \\
& \sum_{A, \alpha} \int \frac{\mathrm{d} \boldsymbol{r} \mathrm{d} \boldsymbol{p}}{(2 \pi \hbar)^{3}} \frac{\delta\left\langle\left\langle 1|\hat{P}| \varrho_{\mathrm{q}}(t)\right\rangle\right\rangle}{\delta\left\langle\left\langle 1\left|\hat{n}_{A \alpha}(x)\right| \varrho(t)\right\rangle\right\rangle}\left(n_{A \alpha}(x)-\left\langle\left\langle 1\left|\hat{n}_{A \alpha}(x)\right| \varrho(t)\right\rangle\right\rangle\right)
\end{aligned}
$$

and has all the properties of a projection operator:

$$
\begin{aligned}
& \mathscr{P}(t) H(\boldsymbol{r}) \quad=H(\boldsymbol{r}), \quad \mathscr{P}(t) \mathscr{P}\left(t^{\prime}\right) \quad=\mathscr{P}(t), \\
& \mathscr{P}(t) n_{A \alpha}(\boldsymbol{r}, \boldsymbol{p})=n_{A \alpha}(\boldsymbol{r}, \boldsymbol{p}), \quad(1-\mathscr{P}(t)) \mathscr{P}(t)=0 .
\end{aligned}
$$

The obtained transport equations have the general meaning and can describe both weakly and strongly nonequilibrium processes of a quantum system with taking into consideration coupled states. In a low density quantum field Boseor Fermi-system the influence of the average value of interaction energy is substantially smaller than the average kinetic energy, and coupled states between the particles are absent. In such a case the set of transport equations $(8.12),(8.13)$ is simplified. It transforms into a kinetic equation [27] in thermo field representation for the average value of the Klimontovich operator $\langle\langle 1|\hat{n}(x)| \varrho(t)\rangle\rangle$ :

$$
\begin{aligned}
& \frac{\partial}{\partial t}\left\langle\left\langle 1\left|\hat{n}_{\boldsymbol{k}}(\boldsymbol{p})\right| \varrho(t)\right\rangle\right\rangle=\left\langle\left\langle 1\left|\dot{\hat{n}}_{\boldsymbol{k}}(\boldsymbol{p})\right| \varrho_{\mathrm{q}}(t)\right\rangle\right\rangle+ \\
& \sum_{\boldsymbol{g}} \int \mathrm{d} \boldsymbol{p}^{\prime} \int_{-\infty}^{t} \mathrm{~d} t^{\prime} \mathrm{e}^{\varepsilon\left(t^{\prime}-t\right)}\left\langle\left\langle J_{n}(\boldsymbol{k} ; t)\left|T\left(t, t^{\prime}\right)\right| \int_{0}^{1} \mathrm{~d} \tau \varrho_{\mathrm{q}}^{\tau}\left(t^{\prime}\right) J_{n}\left(\boldsymbol{g} ; t^{\prime}\right) \varrho_{\mathrm{q}}^{1-\tau}\left(t^{\prime}\right)\right\rangle\right\rangle b_{-\boldsymbol{g}}\left(\boldsymbol{p}^{\prime} ; t^{\prime}\right) .
\end{aligned}
$$

Using the projection operators method, this equation was obtained in [24].

In the next step we will construct such annihilation and creation superoperators, for which the quasiequilibrium thermo vacuum state vector is a vacuum state. Analysing the structure of quasiequilibrium statistical superoperator (8.8), 
one can mark out some part which would correspond to the system of noninteracting quantum $A$-particles. Let us write $\hat{\varrho}_{\mathrm{q}}(t)$ in an evident form and separate terms which are connected with the interaction energy between the particles:

$$
\begin{aligned}
& \hat{\varrho}_{\mathrm{q}}(t)=\exp \left\{-\Phi^{*}(t)-\int \mathrm{d} \boldsymbol{r} \beta(\boldsymbol{r} ; t) \times\right. \\
& \left.\sum_{A, \alpha} \int \frac{\mathrm{d} \boldsymbol{r} \mathrm{d} \boldsymbol{p}}{(2 \pi \hbar)^{3}}\left[\frac{\boldsymbol{p}^{2}}{2 m_{A}} \hat{n}_{A \alpha}(x)-\mu_{A \alpha}(x ; t) \hat{n}_{A \alpha}(x)\right]-\int \mathrm{d} \boldsymbol{r} \beta(\boldsymbol{r} ; t) \hat{H}_{\mathrm{int}}(\boldsymbol{r})\right\} .
\end{aligned}
$$

Using operator equality ( $A$ and $B$ are some operators)

$$
\mathrm{e}^{A+B}=\left[1+\int_{0}^{1} \mathrm{~d} \tau \mathrm{e}^{\tau(A+B)} B \mathrm{e}^{-\tau(A+B)}\right] \mathrm{e}^{A},
$$

the relation for $\hat{\varrho}_{\mathrm{q}}(t)$ can be rewritten in the following form:

$$
\hat{\varrho}_{\mathrm{q}}(t)=\left[1-\int \mathrm{d} \boldsymbol{r} \beta(\boldsymbol{r} ; t) \int_{0}^{1} \mathrm{~d} \tau \hat{\varrho}_{\mathrm{q}}^{\tau}(t) \hat{H}_{\mathrm{int}}(\boldsymbol{r})\left(\hat{\varrho}_{\mathrm{q}}(t)\right)^{-\tau}\right] \hat{\varrho}_{\mathrm{q}}^{0}(t),
$$

where

$$
\begin{aligned}
& \hat{\varrho}_{\mathrm{q}}^{0}(t)=\exp \left\{\Phi(t)-\int \mathrm{d} \boldsymbol{r} \beta(\boldsymbol{r} ; t) \times\right. \\
& \left.\sum_{A, \alpha} \int \frac{\mathrm{d} \boldsymbol{r} \mathrm{d} \boldsymbol{p}}{(2 \pi \hbar)^{3}}\left[\frac{p^{2}}{2 m_{A}} \hat{n}_{A \alpha}(x)-\mu_{A \alpha}(x ; t) \hat{n}_{A \alpha}(x)\right]\right\},
\end{aligned}
$$

or

$$
\hat{\varrho}_{\mathrm{q}}^{0}(t)=\exp \left\{\Phi(t)-\int \mathrm{d} \boldsymbol{r} \beta(\boldsymbol{r} ; t) \sum_{A, \alpha} \int \frac{\mathrm{d} \boldsymbol{p}}{(2 \pi \hbar)^{3}} b_{A \alpha}(x ; t) \hat{n}_{A \alpha}(x)\right\}
$$

where $b_{A \alpha}(x ; t)=\left[\frac{\boldsymbol{p}^{2}}{2 m_{A}} \hat{n}_{A \alpha}(x)-\mu_{A \alpha}(x ; t) \hat{n}_{A \alpha}(x)\right]$. Quasiequilibrium statistical superoperator $\hat{\varrho}_{\mathrm{q}}^{0}(t)$ is bilinear on annihilation and creation superoperators $\hat{a}_{A \alpha}(\boldsymbol{P})$ and $\hat{a}_{A \alpha}^{+}(\boldsymbol{P})$, as well as on the non-perturbed part of Hamiltonian $\bar{H}_{0}$. One can write the total quasiequilibrium superoperator as some non-perturbed part of $\hat{\varrho}_{\mathrm{q}}^{0}(t)$ and the part which describes interaction of quantum particles in the quasiequilibrium state. Further, we introduce the following designation:

$$
\hat{\varrho}_{\mathrm{q}}(t)=\hat{\varrho}_{\mathrm{q}}^{0}(t)+\hat{\varrho}_{\mathrm{q}}^{\prime}(t),
$$

where

$$
\hat{\varrho}_{\mathrm{q}}^{\prime}(t)=-\int \mathrm{d} \boldsymbol{r} \beta(\boldsymbol{r} ; t) \int_{0}^{1} \mathrm{~d} \tau \hat{\varrho}_{\mathrm{q}}^{\tau}(t) \hat{H}_{\mathrm{int}}(\boldsymbol{r})\left(\hat{\varrho}_{\mathrm{q}}(t)\right)^{-\tau} \hat{\varrho}_{\mathrm{q}}^{0}(t) .
$$


Quasiequilibrium thermo vacuum states $\left.\left|\hat{\varrho}_{\mathrm{q}}(t)\right\rangle\right\rangle$ and $\left.\left|\hat{\varrho}_{\mathrm{q}}^{0}(t)\right\rangle\right\rangle$ are not vacuum states for annihilation and creation superoperators $\hat{a}_{A \alpha}(\boldsymbol{P}), \hat{a}_{A \alpha}^{+}(\boldsymbol{P}), \tilde{a}_{A \alpha}(\boldsymbol{P}), \tilde{a}_{A \alpha}^{+}(\boldsymbol{P})$. But for $\left.\left|\hat{\varrho}_{q}^{0}(t)\right\rangle\right\rangle$ one can construct new superoperators $\hat{\gamma}_{A \alpha}(\boldsymbol{P}), \hat{\gamma}_{A \alpha}^{+}(\boldsymbol{P}), \tilde{\gamma}_{A \alpha}(\boldsymbol{P})$, $\tilde{\gamma}_{A \alpha}^{+}(\boldsymbol{P})$ as a linear combination of superoperators $\hat{a}_{A \alpha}(\boldsymbol{P}), \hat{a}_{A \alpha}^{+}(\boldsymbol{P})$ and $\tilde{a}_{A \alpha}(\boldsymbol{P})$, $\tilde{a}_{A \alpha}^{+}(\boldsymbol{P})$ in order to satisfy the conditions:

$$
\begin{aligned}
& \left.\hat{\gamma}_{A \alpha}(\boldsymbol{P} ; t)\left|\varrho_{\mathrm{q}}^{0}(t)\right\rangle\right\rangle=0, \quad\left\langle\langle 1| \hat{\gamma}_{A \alpha}^{+}(\boldsymbol{P} ; t)=0,\right. \\
& \left.\tilde{\gamma}_{A \alpha}(\boldsymbol{P} ; t)\left|\varrho_{\mathrm{q}}^{0}(t)\right\rangle\right\rangle=0, \quad\left\langle\langle 1| \tilde{\gamma}_{A \alpha}^{+}(\boldsymbol{P} ; t)=0 .\right.
\end{aligned}
$$

To achieve this let us consider an action of annihilation superoperators $\hat{a}_{A \alpha}(\boldsymbol{P} ; t)$, $\tilde{a}_{A \alpha}(\boldsymbol{P} ; t)$ on quasiequilibrium state $\left.\left|\varrho_{\mathrm{q}}^{0}\left(t_{0}\right)\right\rangle\right\rangle$ :

$$
\begin{aligned}
& \left.\left.\hat{a}_{A \alpha}(\boldsymbol{P} ; t)\left|\varrho_{\mathrm{q}}^{0}\left(t_{0}\right)\right\rangle\right\rangle=f_{A \alpha}\left(\boldsymbol{P} ; t-t_{0}\right) \tilde{a}_{A \alpha}^{+}(\boldsymbol{P} ; t)\left|\varrho_{\mathrm{q}}^{0}\left(t_{0}\right)\right\rangle\right\rangle, \\
& \left.\left.\tilde{a}_{A \alpha}(\boldsymbol{P} ; t)\left|\varrho_{\mathrm{q}}^{0}\left(t_{0}\right)\right\rangle\right\rangle=\sigma f_{A \alpha}\left(\boldsymbol{P} ; t-t_{0}\right) \hat{a}_{A \alpha}^{+}(\boldsymbol{P} ; t)\left|\varrho_{\mathrm{q}}^{0}\left(t_{0}\right)\right\rangle\right\rangle,
\end{aligned}
$$

where superoperators $\hat{a}_{A \alpha}(\boldsymbol{p} ; t), \hat{a}_{A \alpha}^{+}(\boldsymbol{p} ; t), \tilde{a}_{A \alpha}(\boldsymbol{p} ; t), \hat{a}_{A \alpha}^{+}(\boldsymbol{p} ; t)$ are in the Heisenberg representation

$$
\begin{array}{lll}
\hat{a}_{A \alpha}(\boldsymbol{P} ; t)=\mathrm{e}^{-\frac{1}{\mathrm{i} \hbar} \bar{H}_{0} t} \hat{a}_{A \alpha}(\boldsymbol{P}) \mathrm{e}^{\mathrm{e} \frac{1}{\mathrm{i} \hbar} \bar{H}_{0} t}, & \tilde{a}_{A \alpha}(\boldsymbol{P} ; t)=\mathrm{e}^{-\frac{1}{\mathrm{i} \hbar} \bar{H}_{0} t} \tilde{a}_{A \alpha}(\boldsymbol{P}) \mathrm{e}^{\frac{1}{\mathrm{i} \hbar} \bar{H}_{0} t}, \\
\hat{a}_{A \alpha}^{+}(\boldsymbol{P} ; t)=\mathrm{e}^{-\frac{1}{\mathrm{i} \hbar} \bar{H}_{0} t} \hat{a}_{A \alpha}^{+}(\boldsymbol{P}) \mathrm{e}^{\frac{1}{\mathrm{i} \hbar} \bar{H}_{0} t}, & \tilde{a}_{A \alpha}^{+}(\boldsymbol{P} ; t)=\mathrm{e}^{-\frac{1}{\mathrm{i} \hbar} \bar{H}_{0} t} \tilde{a}_{A \alpha}^{+}(\boldsymbol{P}) \mathrm{e}^{\frac{1}{\mathrm{i} \hbar} \bar{H}_{0} t},
\end{array}
$$

and satisfy commutation relations:

$$
\begin{aligned}
{\left[\hat{a}_{A \alpha}(\boldsymbol{P} ; t), \hat{a}_{B \beta}^{+}\left(\boldsymbol{P}^{\prime} ; t\right)\right]_{\sigma} } & =\delta_{A, B} \delta_{\alpha, \beta} \delta\left(\boldsymbol{P}-\boldsymbol{P}^{\prime}\right), \\
{\left[\tilde{a}_{A \alpha}(\boldsymbol{P} ; t), \tilde{a}_{B \beta}^{+}\left(\boldsymbol{P}^{\prime} ; t\right)\right]_{\sigma} } & =\delta_{A, B} \delta_{\alpha, \beta} \delta\left(\boldsymbol{P}-\boldsymbol{P}^{\prime}\right), \\
{\left[\hat{a}_{A \alpha}(\boldsymbol{P} ; t), \tilde{a}_{B \beta}\left(\boldsymbol{P}^{\prime} ; t\right)\right]_{\sigma} } & =\left[\hat{a}_{A \alpha}^{+}(\boldsymbol{P} ; t), \tilde{a}_{B \beta}^{+}\left(\boldsymbol{P}^{\prime} ; t\right)\right]_{\sigma}=0 .
\end{aligned}
$$

It is necessary to note that superoperators $\hat{H}(\boldsymbol{r}), \hat{n}_{A \alpha}(x)$ are built on superoperators $\hat{a}_{A \alpha}\left(\boldsymbol{p}+\frac{\boldsymbol{q}}{2}\right), \hat{a}_{A \alpha}^{+}\left(\boldsymbol{p}-\frac{\boldsymbol{q}}{2}\right), \tilde{a}_{A \alpha}\left(\boldsymbol{p}+\frac{\boldsymbol{q}}{2}\right), \tilde{a}_{A \alpha}^{+}\left(\boldsymbol{p}-\frac{\boldsymbol{q}}{2}\right)$. Therefore, for convenience here a unit denotion was introduced for arguments like $\boldsymbol{P}=\boldsymbol{p} \pm \frac{\boldsymbol{q}}{2}$. This should be taken into account in further calculations where obvious expressions are needed.

According to general relations of section 6 (6.7)-(6.19), we can introduce new operators $\hat{\gamma}_{A \alpha}(\boldsymbol{P} ; t), \hat{\gamma}_{A \alpha}^{+}(\boldsymbol{P} ; t), \tilde{\gamma}_{A \alpha}(\boldsymbol{P} ; t), \tilde{\gamma}_{A \alpha}^{+}(\boldsymbol{P} ; t)$ via superoperators $\hat{a}_{A \alpha}(\boldsymbol{P} ; t)$, $\hat{a}_{A \alpha}^{+}(\boldsymbol{P} ; t), \tilde{a}_{A \alpha}(\boldsymbol{P} ; t), \tilde{a}_{A \alpha}^{+}(\boldsymbol{P} ; t)$ :

$$
\begin{aligned}
& \hat{\gamma}_{A \alpha}(\boldsymbol{P} ; t)=\sqrt{1+\sigma n_{A \alpha}\left(\boldsymbol{P} ; t, t_{0}\right)}\left[\hat{a}_{A \alpha}(\boldsymbol{P} ; t)-\frac{n_{A \alpha}\left(\boldsymbol{P} ; t, t_{0}\right)}{1+\sigma n_{A \alpha}\left(\boldsymbol{P} ; t, t_{0}\right)} \tilde{a}_{A \alpha}^{+}(\boldsymbol{P} ; t)\right], \\
& \tilde{\gamma}_{A \alpha}^{+}(\boldsymbol{P} ; t)=\sqrt{1+\sigma n_{A \alpha}\left(\boldsymbol{P} ; t, t_{0}\right)}\left[\tilde{a}_{A \alpha}^{+}(\boldsymbol{P} ; t)-\sigma \hat{a}_{A \alpha}(\boldsymbol{P} ; t)\right] .
\end{aligned}
$$

Relations (8.28) satisfy conditions (8.26). Here

$$
\begin{aligned}
n_{A \alpha}\left(\boldsymbol{p}, \boldsymbol{q} ; t, t_{0}\right)=n_{A \alpha}\left(\boldsymbol{P} ; t, t_{0}\right),= & \left\langle\left\langle 1\left|\tilde{a}_{A \alpha}^{+}(\boldsymbol{P} ; t) \tilde{a}_{A \alpha}(\boldsymbol{P} ; t)\right| \varrho_{\mathrm{q}}^{0}\left(t_{0}\right)\right\rangle\right\rangle= \\
& \left\langle\left\langle 1\left|\tilde{a}_{A \alpha}^{+}\left(\boldsymbol{p}-\frac{\boldsymbol{q}}{2} ; t\right) \tilde{a}_{A \alpha}\left(\boldsymbol{p}+\frac{\boldsymbol{q}}{2} ; t\right)\right| \varrho_{\mathrm{q}}^{0}\left(t_{0}\right)\right\rangle\right\rangle,
\end{aligned}
$$

is a quasiequilibrium distribution function of $A$-particle coupled states in momentum space $\boldsymbol{p}, \boldsymbol{q}$, which is calculated with the help of quasiequilibrium thermo 
vacuum state vector $\left.\left.\varrho_{\mathrm{q}}^{0}\left(t_{0}\right)\right\rangle\right\rangle(8.23)$. Function $f_{A \alpha}\left(\boldsymbol{P} ; t-t_{0}\right)$ in formulae (8.27) is connected with $n_{A \alpha}\left(\boldsymbol{P} ; t, t_{0}\right)$ by the relation

$$
f_{A \alpha}\left(\boldsymbol{P} ; t-t_{0}\right)=\frac{n_{A \alpha}\left(\boldsymbol{P} ; t, t_{0}\right)}{1+\sigma n_{A \alpha}\left(\boldsymbol{P} ; t, t_{0}\right)} .
$$

Superoperators $\hat{\gamma}_{A \alpha}(\boldsymbol{P} ; t)$ and $\tilde{\gamma}_{A \alpha}(\boldsymbol{P} ; t), \hat{\gamma}_{A \alpha}^{+}(\boldsymbol{P} ; t)$ and $\tilde{\gamma}_{A \alpha}^{+}(\boldsymbol{P} ; t)$ satisfy the "canonical" commutation relations:

$$
\begin{aligned}
& {\left[\hat{\gamma}_{A \alpha}(\boldsymbol{P} ; t), \hat{\gamma}_{B \beta}^{+}\left(\boldsymbol{P}^{\prime} ; t\right)\right]_{\sigma}=\delta_{A, B} \delta_{\alpha, \beta} \delta\left(\boldsymbol{P}-\boldsymbol{P}^{\prime}\right),} \\
& {\left[\tilde{\gamma}_{A \alpha}(\boldsymbol{P} ; t), \tilde{\gamma}_{B \beta}^{+}\left(\boldsymbol{P}^{\prime} ; t\right)\right]_{\sigma}=\delta_{A, B} \delta_{\alpha, \beta} \delta\left(\boldsymbol{P}-\boldsymbol{P}^{\prime}\right),} \\
& {\left[\hat{\gamma}_{A \alpha}(\boldsymbol{P} ; t), \tilde{\gamma}_{B \beta}\left(\boldsymbol{P}^{\prime} ; t\right)\right]_{\sigma}=\left[\hat{\gamma}_{A \alpha}^{+}(\boldsymbol{P} ; t), \tilde{\gamma}_{B \beta}^{+}\left(\boldsymbol{P}^{\prime} ; t\right)\right]_{\sigma}=0 .}
\end{aligned}
$$

Inversed transformations to superoperators $\hat{a}_{A \alpha}(\boldsymbol{P} ; t), \tilde{a}_{A \alpha}^{+}(\boldsymbol{P} ; t)$ are easily obtained from (8.28):

$$
\begin{aligned}
& \hat{a}_{A \alpha}(\boldsymbol{P} ; t)=\sqrt{1+\sigma n_{A \alpha}\left(\boldsymbol{P} ; t, t_{0}\right)}\left[\hat{\gamma}_{A \alpha}(\boldsymbol{P} ; t)+\frac{n_{A \alpha}\left(\boldsymbol{P} ; t, t_{0}\right)}{1+\sigma n_{A \alpha}\left(\boldsymbol{P} ; t, t_{0}\right)} \tilde{\gamma}_{A \alpha}^{+}(\boldsymbol{P} ; t)\right], \\
& \tilde{a}_{A \alpha}^{+}(\boldsymbol{P} ; t)=\sqrt{1+\sigma n_{A \alpha}\left(\boldsymbol{P} ; t, t_{0}\right)}\left[\tilde{\gamma}_{A \alpha}^{+}(\boldsymbol{P} ; t)+\sigma \hat{\gamma}_{A \alpha}(\boldsymbol{P} ; t)\right] .
\end{aligned}
$$

$\hat{\gamma}_{A \alpha}(\boldsymbol{P} ; t), \hat{\gamma}_{A \alpha}^{+}(\boldsymbol{P} ; t), \tilde{\gamma}_{A \alpha}(\boldsymbol{P} ; t), \tilde{\gamma}_{A \alpha}^{+}(\boldsymbol{P} ; t)$ could be defined as some operators of annihilation and creation of $A$-quasiparticle coupled states, for which quasiequilibrium thermo vacuum state $\left.\left|\varrho_{q}^{0}\left(t_{0}\right)\right\rangle\right\rangle(8.23)$ is a vacuum state. In such a way, we obtained relations of dynamical reflection of superoperators $\hat{a}_{A \alpha}(\boldsymbol{P} ; t), \hat{a}_{A \alpha}^{+}(\boldsymbol{P} ; t)$, $\tilde{a}_{A \alpha}(\boldsymbol{P} ; t), \tilde{a}_{A \alpha}^{+}(\boldsymbol{P} ; t)$ to new superoperators of "quasiparticles" $\hat{\gamma}_{A \alpha}(\boldsymbol{P} ; t), \hat{\gamma}_{A \alpha}^{+}(\boldsymbol{P} ; t)$, $\tilde{\gamma}_{A \alpha}(\boldsymbol{P} ; t), \tilde{\gamma}_{A \alpha}^{+}(\boldsymbol{P} ; t)$.

A set of transport equations (8.12), (8.13) together with dynamical reflections (8.28), (8.30) of superoperators in the thermo field space constitute the basis for a consistent description of the kinetics and hydrodynamics of a dense quantum system with strongly coupled states. Both strongly and weakly nonequilibrium processes of a nuclear matter can be investigated using this approach, in which the particle interaction is characterized by strongly coupled states, taking into account theirs nuclear nature $[1,2,8,9]$.

It is much sequential to describe investigations of kinetic and hydrodynamic processes of a nuclear matter on the basis of quark-gluon interaction. The quantum relativistic theory of kinetic and hydrodynamic processes has its own problems and experiences its impetuous formation [1-6]. In the next section we consider one of the possible ways of describing the kinetics and hydrodynamics of a quark-gluon plasma.

\section{Thermo field transport equations for a quark-gluon plasma}

Investigation of the nonequilibrium properties of a quark-gluon plasma - QGP which can be created after ultrarelativistic collisions of heavy nuclei [1-10] or laser thermonuclear synthesis is topical from the point of view based on the statistical 
approach $[8,9,14,15,48-55]$. Thus, it is important to construct kinetic and hydrodynamic equations for QGP because such a state of a nuclear matter is characterized by high temperature, large density and strong interactions between quarks and gluons which are described by chromodynamics [51-53]. At present there are certain achievements in this direction. The classical theory of transport processes in QGP, based on the relativistic Vlasov-Boltzmann equation, was proposed by Heinz $[54,55]$. On the basis of these works, transport coefficients for weakly nonequilibrium QGP were studied in [56-58]. The Lenard-Balescu-type collision integral for the classical model of a quark plasma was obtained in [59] using the Klimontovich method [60,61]. Hydrodynamics of QGP is considered in papers $[49,50,62,63]$. The Vlasov-Boltzmann equations, like the quantum kinetic ones for Wigner distribution functions for quarks and gluons, were obtained in [49,64-66]. Some interesting results were obtained in paper [67] where the temperature behaviour of the kinetic coefficients of a gluon gas had been studied using the Green-Kubo formalism and $\Phi^{4}-$ model.

However, it is necessary to note that the Vlasov-Boltzmann kinetic equation is correct for a rarefied plasma only (small densities). Thus, it is only the first step in the investigation of transport processes of QGP.

In a dense high temperature quark-gluon plasma, which is characterized by strong interactions, kinetic and hydrodynamic processes are mutually connected and should be considered consistently. In this section we carry out a consistent description of the kinetics and hydrodynamics for QGP on the basis of a nonequilibrium thermo field dynamics using the method of nonequilibrium statistical operator $[27,33,34]$. We will obtain generalized relativistic quantum transport equations of the consistent description of the kinetics and hydrodynamics for QGP. It should be also noted that problems of the description of nonequilibrium properties of QGP were considered in papers [29,68,69].

Consider QGP with the Lagrangian from quantum hydrodynamics [51-53],

$$
\begin{aligned}
L & =\frac{1}{4} F_{\mu \nu}^{a} F_{\mu \nu}^{a}+\Psi^{+}\left(\mathrm{i} \partial^{\mu}+\frac{g}{2} \lambda^{a} A_{\mu}^{a} \gamma_{\mu}\right) \Psi, \\
F_{\mu \nu}^{a} & =\partial_{\mu} A_{\nu}^{a}-\partial_{\nu} A_{\mu}^{a}+g f^{a b c} A_{\mu}^{b} A_{\nu}^{c},
\end{aligned}
$$

where the fields of the matter are spinor quark fields $\Psi$ for which one employs the collective designation $\Psi(x)$ with components $\Psi_{\alpha}^{f_{i}}$, where $i=1,2,3$ (index of colour: red, green, yellow); $f=1, \ldots, 6$ (flavour indices: $b, c, d, s, t, u) ; \alpha=1, \ldots, 4$ (spinor indices); $A_{\nu}^{a}$ are gauge vector fields (Jang-Mill's fields) that correspond to gluons; $a=1, \ldots, 8$ indices of colour; $\lambda^{a}$ means eight Gell-Mann matrices satisfying the commutation relations

$$
\left[\frac{\lambda^{a}}{2}, \frac{\lambda^{b}}{2}\right]=\mathrm{i} f a b c \frac{\lambda^{c}}{2}
$$

$f^{a b c}$ are the structural constants of groups SU(3); $g$ is the gauge constant of connection; $\partial_{\mu}=\partial / \partial x_{\mu}, x_{\mu}=\left(x^{0}=c t, \boldsymbol{x}\right) ; \gamma_{\mu}$ are Dirac matrices [51-53].

The nonequilibrium state of such a system is described by a relativistic quantum nonequilibrium statistical operator $\varrho(t)$ which satisfies the relativistic Liouville 
equation admitting an obviously covariant form. Such a Liouville equation for a thermal quantum field system in a covariant form in the interaction representation was written down in $[67,70]$ on the basis of the Tomonaga-Schwinger equation $[71,72]$ in the next form:

$$
\frac{\delta}{\delta \sigma(x)} \varrho_{\mathrm{int}}(\sigma)-\frac{1}{\mathrm{i} \hbar}\left[H_{\mathrm{int}}(x), \varrho_{\mathrm{int}}(\sigma)\right]=0,
$$

where the nonequilibrium statistical operator $\varrho(\sigma)$ is defined on an arbitrary spacelike surface $\sigma(x)$ [71-73]. When the surface $\sigma(x)$ tends to the plane $t=$ const, equation (9.2) transforms into the quantum Liouville equation in the interaction representation,

$$
\frac{\partial}{\partial t} \varrho_{\text {int }}(t)-\frac{1}{\mathrm{i} \hbar}\left[H_{\text {int }}(x), \varrho_{\text {int }}(t)\right]=0,
$$

where $\varrho_{\text {int }}(t)$ is a nonequilibrium statistical operator in the interaction representation, given on the plane $t=$ const:

$$
H_{\text {int }}(t)=\exp \left\{-\frac{1}{\mathrm{i} \hbar} H_{0} t\right\} H_{\text {int }} \exp \left\{\frac{1}{\mathrm{i} \hbar} H_{0} t\right\}, \quad \varrho_{\text {int }}(t)=\exp \left\{-\frac{1}{\mathrm{i} \hbar} H_{0} t\right\} \varrho(t),
$$

$H_{0}$ and $H_{\text {int }}$ are noninteracting and interacting parts, correspondingly, of the total Hamiltonian $H$ of the system. The Hamiltonian of a quark-gluon system corresponding to the Lagrangian (9.1) was obtained in [74] using the Coulomb gauge. We will represent it in the following form:

$$
\begin{aligned}
H= & H_{0}+H_{\mathrm{int}}, \\
H_{0}= & \frac{1}{2} P_{l}^{a} P_{l}^{a}+\frac{1}{2} \partial_{n} A_{l}^{a} \partial_{n} A_{l}^{a}, \\
H_{\mathrm{int}}= & g f^{a b c} \partial_{l} A_{n}^{a} A_{l}^{b} A_{n}^{c}+\frac{g^{2}}{2} f^{a b c} f^{a d e} A_{l}^{b} A_{n}^{c} A_{l}^{d} A_{n}^{e}-\frac{1}{2} A_{0}^{a} \Delta A_{0}^{a}+ \\
& \Psi^{+}\left[\left(\mathrm{i} \partial_{l}+\frac{g}{2} \lambda^{a} A_{l}^{a}\right) \gamma_{l}+m-\frac{g}{2} \lambda^{a} A_{0}^{a} \gamma_{0}\right] \Psi,
\end{aligned}
$$

selecting the "free field" Hamiltonian $H_{0}$ and the one which describes an interaction between quark and gluon fields. Here $m$ is a colour independent mass matrix for quark flavour indices, $P_{l}^{a}$ means a canonical momentum conjugated to gluon field $A_{l}^{a}$, while zeroth components $A_{0}^{a}$, like in electrodynamics, are not independent and should be inserted in $H_{\text {int }}$ after the solution of the equation of motion:

$$
\Delta A_{0}^{a}=g f^{a b c} A_{l}^{b}\left(P_{l}^{c}+\partial_{l} A_{0}^{c}\right)+\frac{g}{2} \Psi^{+} \gamma_{0} \lambda^{a} \Psi .
$$

On the contrary, proceeding from electrodynamics, it is possible only in the form of infinite series, so that Hamiltonian (9.3) really consists of an infinite number of vertices.

To solve the relativistic Liouville equation (9.2), boundary conditions should be set. Let us search the solutions which depend on time through a certain set 
of observable quantities only, the number of which is sufficient for the description of the nonequilibrium state of a system, using the method of a nonequilibrium statistical operator. For this purpose, we introduce the infinitesimal source $-\eta\left(\varrho_{\text {int }}(\sigma)-\varrho_{\mathrm{q}}^{\text {int }}(\sigma)\right)$ in the right-hand side of equation (9.2), which corresponds to the boundary condition $\varrho_{\text {int }}(\sigma) \rightarrow \varrho_{\mathrm{q}}^{\text {int }}(\sigma)$ with $\sigma \rightarrow-\infty$ and, according to the formalism of nonequilibrium thermo field dynamics, write down this equation in the thermo field representation:

$$
\left.\left.\left.\left.\frac{\delta}{\delta \sigma(x)}\left|\varrho_{\text {int }}(\sigma)\right\rangle\right\rangle-\frac{1}{\mathrm{i} \hbar} \bar{H}_{\mathrm{int}}(x)\left|\varrho_{\mathrm{int}}(\sigma)\right\rangle\right\rangle=-\eta\left(\left|\varrho_{\mathrm{int}}(\sigma)\right\rangle\right\rangle-\left|\varrho_{\mathrm{q}}^{\mathrm{int}}(\sigma)\right\rangle\right\rangle\right),
$$

where the source selects retarded solutions with respect to a reduced description of the nonequilibrium state of a system. $\left.\left|\varrho_{\mathrm{q}}^{\text {int }}(\sigma)\right\rangle\right\rangle$ is a quasi-equilibrium state-vector; $\bar{H}_{\text {int }}(x)$ is the Hamiltonian of interaction between the quark and gluon superfields,

$$
\bar{H}_{0}(x)=\hat{H}_{0}(x)-\tilde{H}_{0}(x), \quad \bar{H}_{\text {int }}(x)=\hat{H}_{\text {int }}(x)-\tilde{H}_{\text {int }}(x),
$$

where $\hat{H}_{0}$ and $\tilde{H}_{0}$ bilinearly depend on Bose superfields $\hat{A}_{\mu}^{a}$ and $\tilde{A}_{\mu}^{a}$ (without and with a tilde line) with corresponding commutation relations analogous to those for the Bose fields [51]; $\hat{H}_{\text {int }}$ and $\tilde{H}_{\text {int }}$ are Hamiltonians composed of gluon Bose superfields $\hat{A}_{\mu}^{a}$ and $\tilde{A}_{\mu}^{a}$, of quark Fermi superfields $\hat{\Psi}^{+}$and $\hat{\Psi}$ without a tilde line, and $\widetilde{\Psi}^{+}$and $\tilde{\Psi}$ with a tilde with corresponding commutation alignments which are similar for both Bose $A_{\mu}^{a}$ and Fermi fields $\Psi[51]$. We shall write "Schrödinger" equation (9.4) in an integral form, introducing

$$
\begin{aligned}
& \left.\left.\left|\varrho_{\text {int }}(\sigma)\right\rangle\right\rangle=\left|\varrho_{\mathrm{q}}^{\mathrm{int}}(\sigma)\right\rangle\right\rangle- \\
& \left.\int_{-\infty}^{\sigma} \mathrm{d}^{4} x^{\prime} \exp \left(\eta \Omega_{\sigma^{\prime} \sigma}\right) T\left(\sigma, \sigma^{\prime}\right)\left(\frac{\delta}{\delta \sigma^{\prime}\left(x^{\prime}\right)}-\frac{1}{\mathrm{i} \hbar} \bar{H}_{\mathrm{int}}\left(x^{\prime}\right)\right)\left|\varrho_{\mathrm{q}}^{\mathrm{int}}\left(\sigma^{\prime}\right)\right\rangle\right\rangle,
\end{aligned}
$$

where $\Omega_{\sigma^{\prime}, \sigma}$ means the volume enclosed between the surface of integration $\sigma^{\prime}\left(x^{\prime}\right)$ and the second one $\sigma(x) ; T\left(\sigma, \sigma^{\prime}\right)$ is an evolution operator,

$$
T\left(\sigma, \sigma^{\prime}\right)=\exp _{+}\left\{\int_{\sigma^{\prime}}^{\sigma} \mathrm{d}^{4} x^{\prime} \frac{1}{\mathrm{i} \hbar} \bar{H}_{\mathrm{int}}\left(\sigma^{\prime}\right)\right\} .
$$

The quasiequilibrium statistical superoperator $\hat{\varrho}_{\mathrm{q}}(\sigma)$ in $\left.\left.\left|\varrho_{\mathrm{q}}(\sigma)\right\rangle\right\rangle=\hat{\varrho}_{\mathrm{q}}(\sigma)|1\rangle\right\rangle$ is defined generally $[27,33,34]$ from the entropy extreme with keeping the normalization and under the conditions that observable quantities $\left\langle p_{n}\right\rangle^{t}=\left\langle\left\langle 1\left|\hat{p}_{n}\right| \varrho(\sigma)\right\rangle\right\rangle$ are given. There are the following characteristic values for QGP: mean density of the quark barion charge $\left\langle b_{\mu}(x)\right\rangle^{t}$, mean densities of the colour currents $\left\langle j_{\mathrm{q} \mu}^{a}(x)\right\rangle^{t}$ for quarks and $\left\langle j_{\mathrm{gl} \mu}^{a}(x)\right\rangle^{t}$ for gluons and also values of the densities of the energymomentum tensor $\left\langle T_{\mu \nu}^{\mathrm{q}}(x)\right\rangle^{t}$ for quarks and $\left\langle T_{\mu \nu}^{\mathrm{gl}}(x)\right\rangle^{t}$ for gluons, in which densities 


$$
\begin{aligned}
b_{\mu}(x), & j_{\mathrm{q} \mu}^{a}(x)+j_{\mathrm{gl} \mu}^{a}(x), T_{\mu \nu}^{\mathrm{q}}(x)+T_{\mu \nu}^{\mathrm{gl}}(x) \text { satisfy the local conservation laws, } \\
& \partial_{\mu} b_{\mu}(x)=0 \\
& \partial_{\mu}\left[j_{\mathrm{q} \mu}^{a}(x)+j_{\mathrm{gl} \mu}^{a}(x)\right]+g f^{a b c} A_{\mu}^{b}(x)\left[j_{\mathrm{q} \mu}^{c}(x)+j_{\mathrm{gl} \mu}^{c}(x)\right]=0 \\
& \partial_{\mu}\left[T_{\mu \nu}^{\mathrm{q}}(x)+T_{\mu \nu}^{\mathrm{gl}}(x)\right]+g\left[j_{\mathrm{q} \mu}^{a}(x)+j_{\mathrm{gl} \mu}^{a}(x)\right] F_{\mu \nu}^{a}(x)=0, \\
& \partial_{\mu} F_{\mu \nu}^{a}(x)+g f^{a b c} A_{\mu}^{b}(x) F_{\mu \nu}^{c}(x)-g\left(j_{\mathrm{q} \nu}^{a}(x)+j_{\mathrm{gl} \nu}^{a}(x)\right)=0 .
\end{aligned}
$$

The densities of operators $b_{\mu}(x), j_{\mathrm{q} \mu}^{a}(x)+j_{\mathrm{gl} \mu}^{a}(x), T_{\mu \nu}^{\mathrm{q}}(x)+T_{\mu \nu}^{\mathrm{gl}}(x)$ are "slowlychanging" and their mean values on the long time scale satisfy the relativistic equations of hydrodynamics; it is necessary to co-ordinate with them the kinetic equations for quark and gluon distribution functions. Local laws (9.6) impose some restrictions on the kinetic processes, and what is more, their role is considerably important at large densities and strong interactions. It shows that the kinetics and hydrodynamics of a quark-gluon system are strongly correlated. That is why, in writing down the kinetic equations for such systems, it is natural to choose the reduced description of the nonequilibrium state in such a way that the proper dynamics of conserved quantities is taken into account automatically.

We introduce Wigner operators for quarks and gluons [50,64,65,75] to obtain the kinetic equations:

$$
f(x ; p)=-\int \frac{\mathrm{d}^{4} y}{(2 \pi \hbar)^{4}} \exp \left\{\frac{1}{\mathrm{i} \hbar} p y\right\} U\left(x, x_{-}\right) \Psi\left(x_{-}\right) \Psi^{+}\left(x_{+}\right) U\left(x_{+}, x\right)
$$

is the Wigner operator of quark density;

$$
\begin{aligned}
G_{\mu \nu}(x ; p)= & \int \frac{\mathrm{d}^{4} y}{(2 \pi \hbar)^{4}} \exp \left\{\frac{1}{\mathrm{i} \hbar} p y\right\} \times \\
& {\left[U\left(x, x_{-}\right) F_{\mu \lambda}\left(x_{-}\right) U\left(x_{-}, x\right)\right] \otimes\left[U\left(x, x_{+}\right) F_{\nu}^{\lambda}\left(x_{+}\right) U\left(x_{+}, x\right)\right] }
\end{aligned}
$$

is the Wigner operator which is connected with the density operator of an energymomentum tensor for gluons:

$$
T_{\mu \nu}^{\mathrm{gl}}(x)=\int \mathrm{d}^{4} p\left\langle\operatorname{Sp} G_{\mu \nu}(x)-\frac{1}{4} g_{\mu \nu} \operatorname{Sp} G_{\lambda}^{\lambda}(x)\right\rangle .
$$

Here $U\left(x^{\prime}, x\right)$ is an operator which was introduced in paper [73],

$$
\begin{aligned}
& U\left(x^{\prime}, x\right)=\exp _{+}\left\{-\frac{g}{\mathrm{i} \hbar c} \int_{x}^{x^{\prime}} \mathrm{d} z^{\mu} A_{\mu}(z)\right\} \\
& x_{-}=x-y / 2, \\
& F_{\mu \nu}=F_{\mu \nu}^{a}(x)\left(\frac{\hbar \lambda_{a}}{2}\right), \\
& x_{+}=x+y / 2, \quad A_{\mu}(x)=A_{\mu}^{a}(x)\left(\frac{\hbar \lambda_{a}}{2}\right) .
\end{aligned}
$$

$U\left(x^{\prime}, x\right)$ is a connection operator, where the integration path $z(s)=x+s\left(x^{\prime}+x\right)$, $0 \leqslant s \leqslant 1$ lies in the plane $t=$ const. The densities of barion charge $b_{\mu}(x)$, quark 
colour current $j_{\mathrm{q} \mu}^{a}(x)$ and energy-momentum tensor $T_{\mu \nu}^{\mathrm{q}}(x)$ are defined via the Wigner operator of quark density, for example,

$$
\begin{aligned}
b_{\mu}(x) & =\int \mathrm{d}^{4} p\left\langle\operatorname{Sp} \gamma_{\mu} f(x ; p)\right\rangle, \\
j_{\mathrm{q} \mu}^{a}(x) & =\int \mathrm{d}^{4} p\left\langle\operatorname{Sp} \frac{\hbar}{2} \lambda_{a} \gamma_{\mu} f(x ; p)\right\rangle, \\
T_{\mu \nu}^{\mathrm{q}}(x) & =\int \mathrm{d}^{4} p\left\langle\operatorname{Sp} \gamma_{\mu} f(x ; p)\right\rangle P_{\nu} .
\end{aligned}
$$

To provide a consistent description of a quark-gluon system, we define the quasiequilibrium statistical superoperator $\left.\left.\hat{\varrho}_{\mathrm{q}}(\sigma), \hat{\varrho}_{\mathrm{q}}(\sigma)|1\rangle\right\rangle=\left|\varrho_{\mathrm{q}}(\sigma)\right\rangle\right\rangle$ in a standard way with keeping to the normalization condition and the following requirements of the averages $[27,33,34]$ :

$$
\begin{array}{ll}
\left\langle\left\langle 1\left|\hat{b}_{\mu}(x)\right| \varrho(\sigma)\right\rangle\right\rangle, & \langle\langle 1|\hat{f}(x ; p)| \varrho(\sigma)\rangle\rangle, \\
\left\langle\left\langle 1\left|\hat{T}_{\mu \nu}(x)\right| \varrho(\sigma)\right\rangle\right\rangle, & \left\langle\left\langle 1\left|\hat{G}_{\mu \nu}(x ; p)\right| \varrho(\sigma)\right\rangle\right\rangle .
\end{array}
$$

Then $\hat{\varrho}_{\mathrm{q}}(\sigma)$ takes the form:

$$
\hat{\varrho}_{\mathrm{q}}(\sigma)=\exp \{-\hat{S}(\sigma)\}
$$

where

$$
\begin{aligned}
& \hat{S}(\sigma)=\Phi^{+}(\sigma)+\int_{\sigma} \mathrm{d} \sigma^{\mu}\left[P^{\nu} \hat{T}_{\mu \nu}-\xi \hat{b}_{\mu}(x)\right]+ \\
& \int_{\sigma} d \sigma^{\mu} \int \mathrm{d}^{4} p\left[a(x ; p) \hat{f}(x ; p)+\omega^{\nu}(x ; p) \hat{G}_{\mu \nu}(x ; p)\right],
\end{aligned}
$$

$\sigma(x)$ is an arbitrary space-like surface passing through point $x: \mathrm{d} \sigma^{\mu}=\mathrm{d} \sigma n^{\mu}$ is a surface element vector; $n^{\mu}$ is a normal vector $\left(\mathrm{d} \sigma^{0}=\mathrm{d}^{3} x\right) ; P^{\nu}(x), \xi(x), a(x ; p)$, $\omega^{\nu}(x ; p)$ are the Lagrange factors that are defined from the self-consistency conditions:

$$
\begin{array}{ll}
\left\langle\left\langle 1\left|\hat{b}_{\mu}(x)\right| \varrho(\sigma)\right\rangle\right\rangle & =\left\langle\left\langle 1\left|\hat{b}_{\mu}(x)\right| \varrho_{q}(\sigma)\right\rangle\right\rangle, \\
\left\langle\left\langle 1\left|\hat{T}_{\mu \nu}(x)\right| \varrho(\sigma)\right\rangle\right\rangle & =\left\langle\left\langle 1\left|\hat{T}_{\mu \nu}(x)\right| \varrho_{q}(\sigma)\right\rangle\right\rangle, \\
\langle\langle 1|\hat{f}(x ; p)| \varrho(\sigma)\rangle\rangle & =\left\langle\left\langle 1|\hat{f}(x ; p)| \varrho_{q}(\sigma)\right\rangle\right\rangle, \\
\left\langle\left\langle 1\left|\hat{G}_{\mu \nu}(x ; p)\right| \varrho(\sigma)\right\rangle\right\rangle=\left\langle\left\langle 1\left|\hat{G}_{\mu \nu}(x ; p)\right| \varrho_{q}(\sigma)\right\rangle\right\rangle,
\end{array}
$$

$P_{\mu}=\beta u_{\mu}, \beta=1 / T(x)$ denotes inverse invariant temperature, $u_{\mu}(x)$ is a local hydrodynamic velocity; $\xi=\beta \mu, \mu_{a}(x)$ is a local chemical potential of $a$-sort quarks $(a: b, c, d, s, t, u)$. Parameters $a(x ; p)$ and $\omega^{\nu}(x ; p)$ are conjugated to averages $\langle\langle 1|\hat{f}(x ; p)| \varrho(\sigma)\rangle\rangle,\left\langle\left\langle 1\left|\hat{G}_{\mu \nu}(x ; p)\right| \varrho(\sigma)\right\rangle\right\rangle . \hat{T}_{\mu \nu}(x), \hat{b}_{\mu}(x), \hat{f}(x ; p), \hat{G}_{\mu \nu}(x ; p)$ are superoperators constructed according to (9.7)-(9.13) and the thermo field dynamics formalism [27], on Bose superfields $\hat{A}_{\mu}^{a}(x)$ and Fermi superfields $\hat{\Psi}^{+}, \hat{\Psi} . \Phi^{+}(\sigma)$ is calculated from the normalization condition: $\left\langle\left\langle 1 \mid \varrho_{q}(\sigma)\right\rangle\right\rangle$ equals to 1 . Accordingly, 
superoperator $\tilde{\varrho}_{\mathrm{q}}^{+}(\sigma)$ is built on operators $\tilde{T}_{\mu \nu}(x), \tilde{b}_{\mu}(x), \tilde{f}(x ; p), \tilde{G}_{\mu \nu}(x ; p)$ which are expressed via Bose superoperators $\tilde{A}_{\mu}^{a}(x)$ and Fermi superoperators $\tilde{\Psi}^{+}, \tilde{\Psi}$ (with a tilda line). Taking into consideration the structure of quasiequilibrium statistical superoperator (9.15) and (9.16), one can write the nonequilibrium thermo vacuum state vector (9.5) in the interaction representation in the following form:

$$
\begin{aligned}
& \left.\left.\left|\varrho_{\text {int }}(\sigma)\right\rangle\right\rangle=\left|\varrho_{\mathrm{q}}^{\mathrm{int}}(\sigma)\right\rangle\right\rangle-\int_{-\infty}^{\sigma} \mathrm{d}^{4} x^{\prime} \mathrm{e}^{\eta \Omega_{\sigma^{\prime} \sigma}} T\left(\sigma, \sigma^{\prime}\right) \times \\
& \left.\left|\int_{0}^{1} \mathrm{~d} \tau \mathrm{e}^{-\tau S^{\mathrm{int}}\left(\sigma^{\prime}\right)}\left\{\frac{\delta}{\delta \sigma^{\prime}\left(x^{\prime}\right)}-\frac{1}{\mathrm{i} \hbar} \bar{H}_{\mathrm{int}}\left(x^{\prime}\right)\right\} S^{\mathrm{int}}\left(\sigma^{\prime}\right) \mathrm{e}^{\tau S^{\mathrm{int}}\left(\sigma^{\prime}\right)} \varrho_{\mathrm{q}}^{\mathrm{int}}\left(\sigma^{\prime}\right)\right\rangle\right\rangle .
\end{aligned}
$$

Using the rule $[71,72] \frac{\delta}{\delta \sigma(x)} \int_{\sigma} \mathrm{d} \sigma^{\mu}\left(x^{\prime}\right) A_{\mu}\left(x^{\prime}\right)=\frac{\partial}{\partial x_{\mu}} A_{\mu}(x)$, for $\left(\delta S^{\text {int }}\left(\sigma^{\prime}\right)\right) /\left(\delta \sigma^{\prime}\left(x^{\prime}\right)\right)$ we obtain

$$
\begin{aligned}
& \frac{\delta}{\delta \sigma^{\prime}\left(x^{\prime}\right)} S^{\mathrm{int}}\left(\sigma^{\prime}\right)=\frac{\partial}{\partial x_{\mu}^{\prime}}\left[P^{\nu} T_{\mu \nu}^{\mathrm{int}}\left(x^{\prime}\right)-\xi b_{\mu}^{\mathrm{int}}\left(x^{\prime}\right)+\right. \\
& \left.\int \mathrm{d}^{4} p\left[a\left(x^{\prime} ; p\right) f^{\mathrm{int}}\left(x^{\prime} ; p\right)+\omega^{\nu}\left(x^{\prime} ; p\right) G_{\mu \nu}^{\mathrm{int}}\left(x^{\prime} ; p\right)\right]\right] .
\end{aligned}
$$

The action of the operator $-\frac{1}{\mathrm{i} \hbar} H_{\mathrm{int}}(x)$ on $S^{\mathrm{int}}\left(\sigma^{\prime}\right)$ reads:

$$
\begin{aligned}
& \frac{1}{\mathrm{i} \hbar} H_{\text {int }}(x) S^{\text {int }}\left(\sigma^{\prime}\right)=-\int_{\sigma^{\prime}} \mathrm{d} \sigma^{\mu}\left(x^{\prime \prime}\right)\left[P^{\nu} \dot{T}_{\mu \nu}^{\mathrm{int}}\left(x^{\prime \prime}\right)-\xi \dot{b}_{\mu}^{\mathrm{int}}\left(x^{\prime \prime}\right)\right]+ \\
& \int_{\sigma^{\prime}} \mathrm{d} \sigma^{\mu}\left(x^{\prime \prime}\right) \int \mathrm{d}^{4} p\left[a\left(x^{\prime \prime} ; p\right) \dot{f}^{\mathrm{int}}\left(x^{\prime \prime} ; p\right)+\omega^{\nu}\left(x^{\prime \prime} ; p\right) \dot{G}_{\mu \nu}^{\mathrm{int}}\left(x^{\prime \prime} ; p\right)\right],
\end{aligned}
$$

where

$$
\begin{aligned}
& \dot{b}_{\mu}^{\mathrm{int}}\left(x^{\prime \prime}\right)=-\frac{1}{\mathrm{i} \hbar}\left[H_{\mathrm{int}}\left(x^{\prime}\right), b_{\mu}^{\mathrm{int}}\left(x^{\prime \prime}\right)\right], \dot{f}^{\mathrm{int}}\left(x^{\prime \prime} ; p\right)=-\frac{1}{\mathrm{i} \hbar}\left[H_{\mathrm{int}}\left(x^{\prime}\right), f^{\mathrm{int}}\left(x^{\prime \prime} ; p\right)\right], \\
& \dot{T}_{\mu \nu}^{\mathrm{int}}\left(x^{\prime \prime}\right)=-\frac{1}{\mathrm{i} \hbar}\left[H_{\mathrm{int}}\left(x^{\prime}\right), T_{\mu \nu}^{\mathrm{int}}\left(x^{\prime \prime}\right)\right], \dot{G}_{\mu \nu}^{\mathrm{int}}\left(x^{\prime \prime} ; p\right)=-\frac{1}{\mathrm{i} \hbar}\left[H_{\mathrm{int}}\left(x^{\prime}\right), G_{\mu \nu}^{\mathrm{int}}\left(x^{\prime \prime} ; p\right)\right] .
\end{aligned}
$$

Using the nonequilibrium thermo vacuum state vector (9.18), we obtain a system of coupled equations for averages (9.14) in the interaction representation:

$$
\begin{array}{r}
p^{\mu} \frac{\partial}{\partial x_{\mu}}\left\langle\left\langle 1\left|\hat{b}_{\mu}(x)\right| \varrho_{\text {int }}(\sigma)\right\rangle\right\rangle=0, \\
p^{\mu} \frac{\partial}{\partial x_{\mu}}\left\langle\left\langle 1\left|\hat{T}_{\mu \nu}(x)\right| \varrho_{\text {int }}(\sigma)\right\rangle\right\rangle=p^{\mu} \frac{\partial}{\partial x_{\mu}}\left\langle\left\langle 1\left|\hat{T}_{\mu \nu}(x)\right| \varrho_{\mathrm{q}}^{\text {int }}(\sigma)\right\rangle\right\rangle+ \\
p^{\mu} \frac{\partial}{\partial x_{\mu}} \int_{-\infty}^{\sigma} \mathrm{d}^{4} x^{\prime} \mathrm{e}^{\eta \Omega_{\sigma^{\prime} \sigma}}\left\langle\left\langle 1\left|\hat{T}_{\mu \nu}(x) T\left(\sigma, \sigma^{\prime}\right)\right| \times\right.\right. \\
\left.\left.\int_{0}^{1} \mathrm{~d} \tau \mathrm{e}^{-\tau S^{\text {int }}\left(\sigma^{\prime}\right)}\left\{\frac{\delta}{\delta \sigma^{\prime}\left(x^{\prime}\right)}-\frac{1}{\mathrm{i} \hbar} H_{\text {int }}\left(x^{\prime}\right)\right\} S^{\text {int }}\left(\sigma^{\prime}\right) \mathrm{e}^{\tau S^{\text {int }}\left(\sigma^{\prime}\right)} \varrho_{\mathrm{q}}^{\text {int }}\left(\sigma^{\prime}\right)\right\rangle\right\rangle,
\end{array}
$$




$$
\begin{array}{r}
p \cdot D(x)\left\langle\left\langle 1|\hat{f}(x, p)| \varrho_{\text {int }}(\sigma)\right\rangle\right\rangle=p \cdot D(x)\left\langle\left\langle 1|\hat{f}(x, p)| \varrho_{\mathrm{q}}^{\text {int }}(\sigma)\right\rangle\right\rangle+ \\
p \cdot D(x) \int_{-\infty}^{\sigma} \mathrm{d}^{4} x^{\prime} \mathrm{e}^{\eta \Omega_{\sigma^{\prime} \sigma}\left\langle\left\langle 1\left|\hat{f}(x, p) T\left(\sigma, \sigma^{\prime}\right)\right| \times\right.\right.} \\
\left.\left.\int_{0}^{1} \mathrm{~d} \tau \mathrm{e}^{-\tau S^{\text {int }}\left(\sigma^{\prime}\right)}\left\{\frac{\delta}{\delta \sigma^{\prime}\left(x^{\prime}\right)}-\frac{1}{\mathrm{i} \hbar} H_{\text {int }}\left(x^{\prime}\right)\right\} S^{\text {int }}\left(\sigma^{\prime}\right) \mathrm{e}^{\tau S^{\text {int }}\left(\sigma^{\prime}\right)} \varrho_{\mathrm{q}}^{\text {int }}\left(\sigma^{\prime}\right)\right\rangle\right\rangle, \\
p \cdot \tilde{D}(x)\left\langle\left\langle 1\left|\hat{G}_{\mu \nu}(x, p)\right| \varrho_{\text {int }}(\sigma)\right\rangle\right\rangle=p \cdot \tilde{D}(x)\left\langle\left\langle 1\left|\hat{G}_{\mu \nu}(x, p)\right| \varrho_{\mathrm{q}}^{\text {int }}(\sigma)\right\rangle\right\rangle+ \\
p \cdot \tilde{D}(x) \int_{-\infty}^{\sigma} \mathrm{d}^{4} x^{\prime} \mathrm{e}^{\eta \Omega_{\sigma^{\prime} \sigma}\left\langle\left\langle 1\left|\hat{G}_{\mu \nu}(x, p) T\left(\sigma, \sigma^{\prime}\right)\right| \times\right.\right.} \\
\left.\left.\int_{0}^{1} \mathrm{~d} \tau \mathrm{e}^{-\tau S^{\operatorname{int}}\left(\sigma^{\prime}\right)}\left\{\frac{\delta}{\delta \sigma^{\prime}\left(x^{\prime}\right)}-\frac{1}{\mathrm{i} \hbar} H_{\text {int }}\left(x^{\prime}\right)\right\} S^{\text {int }}\left(\sigma^{\prime}\right) \mathrm{e}^{\tau S^{\text {int }}\left(\sigma^{\prime}\right)} \varrho_{\mathrm{q}}^{\text {int }}\left(\sigma^{\prime}\right)\right\rangle\right\rangle,
\end{array}
$$

while the space-like surface $\sigma(x)$ tends to the plane $t=$ const. Here $D(x)$ and $\tilde{D}(x)$ are covariant derivatives. They act on the Wigner operators of quarks and gluons, correspondingly:

$$
D_{\mu}(x) f(x, p) \equiv\left(\frac{\partial}{\partial x_{\mu}}+\frac{1}{g} \mathrm{i} \hbar c A_{\mu}(x)\right) f(x, p),
$$

where $A_{\mu}(x)=A_{\mu}^{a}(x) T_{a}$ with $\left(T_{a}\right)_{b c}=-\mathrm{i} \hbar f_{a b c}$ is an $8 \times 8$ matrix. The tildian covariant derivative acts on the Wigner operator of gluons

$$
\tilde{D}_{\alpha}(x) G_{\mu \nu}(x, p) \equiv \frac{\partial}{\partial x_{\alpha}} G_{\mu \nu}(x, p) \frac{g}{\mathrm{i} \hbar c}\left[A_{\alpha}, G_{\mu \nu}(x, p)\right]
$$

with a commutator between two $8 \times 8$ matrices $\left(A_{\alpha}\right)^{m n}=A_{\alpha}^{a}\left(T_{a}\right)^{m n}$ and $\left(\hat{G}_{\mu \nu}\right)^{a b}$. An action of these covariant derivatives on the Wigner operator of quarks $p$. $D(x) f(x, p)$ and on the Wigner operator of gluons $p \cdot \tilde{D}(x) \hat{G}_{\mu \nu}(x, p)$ was calculated in papers $[64,65]$. The set of equations (9.19)-(9.22) without taking into account the barion charge transport $\left\langle\left\langle 1\left|\hat{b}_{\mu}(x)\right| \varrho(t)\right\rangle\right\rangle$ and the total momentum tensor $\left\langle\left\langle 1\left|\hat{T}_{\mu}(x)\right| \varrho(t)\right\rangle\right\rangle$ turns to a connected system of kinetic equations for the average values of Wigner operators of quarks $\langle\langle 1|\hat{f}(x, p)| \varrho(t)\rangle\rangle$, gluons $\left\langle\left\langle 1\left|\hat{G}_{\mu}(x, p)\right| \varrho(t)\right\rangle\right\rangle$. It generalizes the results of papers [64-66].

We have obtained a system of coupled relativistic transport equations of a consistent description of the kinetics and hydrodynamics for QGP in thermo field representation. This system of equations is strongly nonlinear and could be used to describe both strongly and weakly nonequilibrium states of a system. However, it should be noted that the following transformations for the use of the system of relativistic transport equations (9.19)-(9.22) have to be made. Since the quasiequilibrium thermo vacuum state vector $\left.\left|\varrho_{\mathrm{q}}(\sigma)\right\rangle\right\rangle$, which is used in averaging equations (9.19)-(9.22), is not a vacuum state vector for Bose superfields 
$\tilde{A}_{\mu}^{a}, \hat{A}_{\mu}^{a}$ of gluons and Fermi superfields $\hat{\Psi}, \hat{\Psi}^{+}, \tilde{\Psi}, \tilde{\Psi}^{+}$of quarks, it is necessary to construct Bose superfields of gluons and Fermi superfields of quarks for which it is a vacuum state vector, as in paper [27]. Such superfields will depend on the parameters of state $P^{\nu}(x), \beta \mu, a(x ; p), \omega^{\nu}(x ; p)$, besides, all the superoperators $\hat{T}_{\mu \nu}(x), \hat{b}_{\mu}(x), \hat{f}(x ; p), \hat{G}_{\mu \nu}(x ; p), \bar{H}_{\text {int }}(x), T\left(\sigma, \sigma^{\prime}\right)$ in a system of relativistic transport equations (9.19)-(9.22) should be expressed in a such way where $\left.\left|\varrho_{\mathrm{q}}(\sigma)\right\rangle\right\rangle$ is the vacuum state vector. Besides, the use of space-like surface $\sigma(x)$ is justified at the calculation of generalized equations in the invariant form. Since the final results do not depend on the choice of surface $\sigma$, one has to rewrite equations (9.19)-(9.22) on the surface directed to the plane $t=$ const. So, it is useful to choose $\eta=\varepsilon V^{-1}$, where $V$ denotes the volume occupied by system [70]. Then $\Omega_{\sigma^{\prime} \sigma}=\left(t^{\prime}-t\right) V$ and $\eta \Omega_{\sigma^{\prime} \sigma}=\varepsilon\left(t^{\prime}-t\right)$. Thus, in transport equations (9.19)-(9.22),

$\int_{-\infty}^{\sigma} \mathrm{d}^{4} x^{\prime} \exp \left(\eta \Omega_{\sigma^{\prime} \sigma}\right) \rightarrow \int_{-\infty}^{t} \mathrm{~d} t^{\prime} \exp \left(\varepsilon\left(t^{\prime}-t\right)\right)$ and superoperators $\hat{\varrho}_{\text {int }}(t), \hat{\varrho}_{\mathrm{q}}^{\text {int }}(t)$, $T\left(t, t^{\prime}\right)$ will be given on the plane $t=$ const. Such questions will be considered in the next paper, in particular, in the investigation of the relativistic transport equations for weakly nonequilibrium QGP.

\section{Conclusions}

In this paper the conception of nonequilibrium thermo field dynamics on the basis of a nonequilibrium statistical operator has been applied to the construction of transport equations of dense quantum systems. Hydrodynamic equations in thermo field representation have been obtained for both strongly and weakly nonequilibrium processes. Transport cores, connected with transport coefficients of viscosity and thermal conductivity, have been defined, too. They are calculated with the help of a quasiequilibrium thermo vacuum state vector. The last one is a vacuum state vector for annihilation and creation superoperators which depend on thermodynamic parameters $F_{n}(t)$ of a system. The consistency of both annihilation and creation superoperators and the corresponding vacuum state is accomplished then by the mentioned approach. In particular, it is achieved by the generalization of the Bogolubov transformations in thermo field dynamics [32] for a nonequilibrium case. General transport equations within nonequilibrium thermo field dynamics - nonequilibrium statistical operator made it possible to obtain an equation of a generalized description of the kinetics and hydrodynamics for a dense quantum system with strongly coupled states. In such a case, $f_{A \alpha}(x ; t)$ - the Wigner function of the $A$-particle coupled state, and the average value of the total energy density operator $\langle\langle 1|\hat{H}(\boldsymbol{r})| \varrho(t)\rangle\rangle$ have been chosen as parameters of a shortened description. Particle annihilation and creation superoperators for the quasiequilibrium thermo vacuum state vector of noninteracting particles were defined in (8.22). It gives us the possibility for the construction of a diagram technique in the calculation of the corresponding transport cores. These investigations and calculations are important for actual nuclear systems $[8,9]$. 
We have considered a method for obtaining generalized transport equations for QGP - one of the nuclear matter states. These equations were obtained in the most general form. To be used, their structure needs a lot of transformations, especially for transport cores. The consistent description of the kinetics and hydrodynamics is based on a set of fundamental parameters of a shortened description: the average values of Wigner operators for quarks and gluons $\langle\langle 1|\hat{f}(x)| \varrho(t)\rangle\rangle,\left\langle\left\langle 1\left|\hat{G}_{\mu \nu}(x)\right| \varrho(t)\right\rangle\right\rangle$ and the average values of the density operator of barion charge $\left\langle\left\langle 1\left|\hat{b}_{\mu}(x)\right| \varrho(t)\right\rangle\right\rangle$ and the total energy-momentum tensor $\left\langle\left\langle 1\left|\hat{T}_{\mu \nu}(x)\right| \varrho(t)\right\rangle\right\rangle$ of quarks and gluons which constitute the basis for the hydrodynamic description. The set of equations obtained permits the investigations of weakly nonequilibrium processes and kinetic equations like the Boltzmann-Vlasov or Lenard-Balescu ones for diluted QGP.

The problem of the investigation of transport coefficients: viscosity and thermal conductivity as well as excitations of QGP still remains. It might be considered in view of the obtained transport equations. This will be the subject of our future work.

\section{References}

1. J.W.Negele. The mean field theory of nuclear structure and dynamics. // Rev. Mod. Phys., 1982, vol. 54, No 4, p. 913-1015.

2. L.McLerran. The physics of the quark-gluon plasma. // Rev. Mod. Phys., 1986, vol. 58, No 4, p. 1021-1064.

3. B.Blättel, V.Koch, U.Mosel. Transport-theoretical analysis of relativistic heavy-ion collisions. // Rep. on Progr. in Phys., 1993, vol. 56, No 1, p. 1-62.

4. S.Mrówczyńsky. In: Quark-qluon plasma. World Scientific, Singapore, 1990.

5. J.Cleymans, R.V.Gavai, E.Suhonen. Quarks and gluons at high temperatures and densities. // Phys. Rep., 1986, vol. 130, No 4, p.

6. M.Jacob, H.Satz. Quark matter formation and heavy ion collisions. World Scientific, Singapore, 1982.

7. L.McLerran. Probes of the quark-gluon plasma as it might be produced in ultrarelativistic nuclear collisions. // Acta Phys. Polon. B, 1985, vol. 16, No 7, p. 669-682.

8. V.D.Toneev, H.Shultz, K.K.Gudima, G.Röpke. Towards study of hot and dense nuclear matter in heavy ion collisions. // Physics of Elementary Particles and Nuclei (Particles \& Nuclei), 1986, vol. 17, No 6, p. 1093-1172 (in Russian).

9. G.Röpke, H.Shultz, K.K.Gudima, V.D.Toneev. Dynamical approaches to heavy-ion collision in intermediate energy region. // Physics of Elementary Particles and Nuclei (Particles \& Nuclei), 1990, vol. 21, No 2, p. 364-418 (in Russian).

10. H.Hora. Physics of lased driven plasmas. John Wiley and Sons, New York, 1981.

11. J.J.Duderstadt, G.A.Moses. Inertial confinement fusion. John Wiley and Sons, New York, 1982.

12. K.A.Brueckner, S.Jorna. In: Report on laser driven fusion, KMS Fusion, Inc. Ann Arbor, Michigan, USA, May 10, 1973. Reprinted in Rev. Mod. Phys., 1994. Russian transl.: Atomizdat, Moscow, 1977 (in Russian).

13. J.Naccols, L.Wood, A.Tissen, G.Zimmermann. Problems of laser thermonuclear synthesis. Atomizdat, Moscow, 1977 (in Russian). 
14. L.V.Keldysh. Diagram technique for nonequilibrium processes. // Zhurn. Exp. Teor. Fiz., 1964, vol. 47, No 4(10), p. 1515-1527 (in Russian).

15. P.Danielewicz. Quantum theory of nonequilibrium processes I. // Ann. Phys., 1984, vol. 152, No 2, p. 239-304. Quantum theory of nonequilibrium processes II. Application to nuclear collisions. // Ann. Phys., 1984, vol. 152, No 2, p. 304-326.

16. W.Boterman, R.Malfliet. Quantum transport theory of nuclear matter. // Phys. Rep., 1990, vol. 198, No 3, p. 115-194.

17. Yu.L.Klimontovich, D.Kremp, W.-D.Kraeft. In: Advances of chemical physics. Wiley, New York, 1987, p. 175.

18. R.E.Snider. A density-corrected quantum Boltzmann equation. // J. Stat. Phys., 1990, vol. 61 , No $1 / 2$, p. 443-465.

19. R.E.Snider. A reinterpretation of dense gas kinetic theory. // J. Stat. Phys., 1991, vol. 63, No $3 / 4$, p. 707-718.

20. V.G.Morozov, G.Röpke. Quantum kinetic equation for nonequilibrium dense systems. // Physica A, 1995, vol. 221, p. 511-538.

21. D.N.Zubarev, V.G.Morozov, G.Röpke. Statistical mechanics of nonequilibrium processes. Vol. 1: Basic concepts, kinetic theory. Academie Verlag, Berlin, 1996.

22. D.N.Zubarev, V.G.Morozov. Formulation of boundary conditions for the Bogolubov hierarhy with allowance for local conservation laws. // Teor. Mat. Fiz., 1984, vol. 60, No 2, p. 270-279 (in Russian).

23. D.N.Zubarev, V.G.Morozov, I.P.Omelyan, M.V.Tokarchuk. On kinetic equations for dense gases and liquids. // Teor. Mat. Fiz., 1991, vol. 87, No 1, p. 113-129 (in Russian).

24. T.Atimitsu. Kinetic equations within the formalism of nonequilibrium thermo field dynamics. // Physica A, 1988, vol. 148, No 3, p. 427-455.

25. T.Arimitsu. Nonequilibrium open systems and nonequilibrium thermo field dynamics. // Physica A, 1989, vol. 158, No 1, p. 317-325.

26. T.Arimitsu. Thermal processes in the hydrodynamics stage in terms of nonequilibrium thermo field dynamics. // J. Phys. A: Math. Gen., 1991, vol. 24, No 24, p. L1415-1421.

27. D.N.Zubarev, M.V.Tokarchuk. Nonequilibrium thermo field dynamics and nonequilibrium statistical operator method. // Teor. Mat. Fiz., 1991, vol. 87, No 2, p. 286-310 (in Russian).

28. T.Arimitsu. A canonical formalism of dissipative quantum systems. Nonequilibrium thermo field dynamics. // Cond. Matt. Phys., 1994, No 4, p. 23-88.

29. P.A.Henning. Thermo field dynamics for quantum fields with continuous mass spectrum. // Phys. Rep., 1995, vol. 253, No 5\&6, p. 235-380.

30. K.Kawasaki, J.D.Gunton. Theory of nonlinear shear viscosity and normal stress effects. // Phys. Rev. A, 1973, vol. 8, No 4, p. 2048-2064.

31. Y.Takahashi, H.Umezawa. Thermo field dynamics. // Collective Phenomena, 1975, vol. 2, No 2, p. 55-80.

32. H.Umezawa, H.Matsumoto, M.Tachiki. Thermo field dynamics and condensed states. North Holland, Amsterdam, 1982.

33. D.N.Zubarev. Nonequilibrium statistical thermodynamics. Consultant Bureau, New York, 1974.

34. D.N.Zubarev. In: Reviews fo science and technology. Modern problems of mathematics, vol. 15. VINITI, Moscow, 1980, p. 131-220 (in Russian).

35. T.Arimitsu, H.Umezawa. General formulation of nonequilibrium thermo field dynamics. // Progr. Theor. Phys. (Progr. Lett.), 1985, vol. 74, No 2, p. 429-432. 
36. T.Arimitsu, H.Umezawa. Nonequilibrium thermo field dynamics. // Progr. Theor. Phys., 1987, vol. 77, No 1, p. 32-52.

37. T.Arimitsu, H.Umezawa. General formulation of nonequilibrium thermo field dynamics. // Progr. Theor. Phys., 1987, vol. 77, No 1, p. 53-67.

38. M.Schmutz. Real-time Green's functions in many body problems. // Z. Phys. B, 1978, vol. 30, No 1, p. 97-107.

39. K.Blum. Density matrix theory and applications. Plenum Press, New-York and London, 1981.

40. H.Umezawa, Y.Yamanaka. Micro, macro and thermal concepts in quantum field theory. // Adv. Phys., 1988, vol. 37, No 5, p. 531-557.

41. T.Arimitsu. Canonical formalism of dissipative fields in thermo field dynamics. // J. Math. Phys., 1987, vol. 28, No 11, p. 2741-2752.

42. H.Matsumoto, H.Umezawa, J.P.Whitehead. Vacuum diagrams in perturbative thermo field dynamics. // Progr. Theor. Phys., 1986, vol. 76, No 1, p. 260-282.

43. A.Hosoya, M.Sakagami, M.Takao. Nonequilibrium thermo field dynamics in field theory: transport coefficients. // Ann. Phys., 1984, vol. 154, p. 229-253.

44. A.A.Abrikosov, L.P.Gorkov, I.E.Dzyaloshinski. Methods of quantum field theory in statistical physics. Dover Publ., New York, 1975.

45. G.Röpke, H.Shulz. Including cluster formation into a Vlasov-Ueling-Uhlenbeck approach to intermediate energy heavy ion collisions. // Nucl. Phys. A, 1988, vol. 477, p. $472-486$.

46. K.Morawetz, G.Röpke. Memory effects and virial corrections in nonequilibrium dense systems. // Phys. Rev. E, 1995, vol. 51, No 5, p. 4246-4261.

47. Th.Bornath, D.Kremp. W.-D.Kraeft, M.Schlanges. Kinetic equations for a nonideal quantum system. // Phys. Rev. E, 1996, vol. 54, No 4, p. 3274-3284.

48. E.V.Shchurjak. Theory of hadron plasma. // JETP, 1978, vol. 74, No 2, p. 408-420 (in Russian).

49. H.-Th.Elze, U.Heinz. Quark-gluon transport theory. // Phys. Rep., 1989, vol. 183, No 3, p. 82-135.

50. D.Vasak, M.Gyulassy, H.-Th.Elze. Quantum transport theory for Abelian plasmas. // Ann. Phys., 1987, vol. 173, p. 462-492.

51. A.I.Akhiezer, S.V.Peletminsky. Theory of fundamental interactions. Kiev, Naukova Dumka, 1993 (in Russian).

52. K.Huang. Quarks, leptons and gauge fields. World Scientific, Singapore, 1982.

53. F.J.Yndurain. Quantum chromodynamics. An Introduction to the theory of quarks and gluons. Springer-Verlag, New York, 1983.

54. U.Heinz. Quark-gluon transport theory. I. The classical theory. // Ann. Phys., 1985, vol. 161 , p. $48-80$.

55. U.Heinz. Quark-gluon transport theory. II. Color response and color correlations in a quark-gluon plasma. // Ann. Phys., 1986, vol. 168, p. 148-180.

56. W.Czyż, W.Florkowski. Kinetic coefficients for quark-antiquark plasma. // Acta Phys. Polon. B, 1986, vol. 17, No 9, p. 819-837.

57. S.Mrówczyński. On the transport coefficients of a quark plasma. // Acta Phys. Polon. B, 1988, vol. 19, No 1, p. 91-96.

58. S.Mrówczyński. Quark-gluon plasma oscilations in transport theory. // Physica A, 1989, vol. 158, No 2, p. 136-145.

59. Yu.A.Markov, M.A.Markova. The Balescu-Lenard collision term for a quark plasma. 
The classical model. // Teor. Mat. Fiz., 1995, vol. 103, No 1, p. 123-137 (in Russian). The classical limit of a quantum kinetic equation for quarks. // Teor. Mat. Fiz., 1996, vol. 108, No 1, p. 159-175 (in Russian).

60. Yu.L.Klimontovich. The statistical theory of nonequilibrium processes in a plasma. Pergamon, Oxford, 1964.

61. Yu.L.Klimontovich. Kinetic theory of nonideal gases and nonideal plasmas. Pergamon, Oxford, 1982; Kinetic theory of electrodynamic processes in plasmas. Springer Verlag, Berlin, Heidelberg, New York, 1982.

62. J.-P.Blaizot, J.-Y.Ollitrautt. Hydrodynamics of a quark-gluon plasma undergoing a phase transition. // Nucl. Phys. A, 1986, vol. 458, p. 745-772.

63. J.-P.Blaizot. Hydrodynamics of quark-gluon plasmas. // Acta Phys. Polon. B, 1987, vol. 18, No 7, p. 659-680.

64. H.-Th.Elze, M.Gyulassy, D.Vasak. Transport equations for the QCD quark Wigner operator. // Phys. Lett. B, 1986, vol. 177, No 3\&4, p. 402-408.

65. H.-Th.Elze, M.Gyulassy, D.Vasak. Transport equations for the QCD quark Wigner operator. // Nucl. Phys. B, 1986, vol. 276, p. 706-728.

66. U.Heinz. Non-equilibrium dynamics in finite-temperature QCD. // Physica A, 1989, vol. 158, No 1, p. 111-124.

67. A.V.Prosorkevich, S.A.Smolyansky. On the theory of quantum relativistic equations. // Teor. Mat. Fiz., 1976, vol 28, No 2, p. 262-266 (in Russian).

68. N.P.Landsman. Non-shell unstable particles in thermal field theory. // Ann. Phys., 1988, vol. 186, p. 141-205.

69. N.P.Landsman. How dissipation solves the infrared problem in thermal QCD. // Physica A, 1989, vol. 158, No 1, p. 200-224.

70. D.N.Zubarev, A.V.Prosorkevich, S.A.Smolyansky. Derivation of nonlinear generalised equations of quantum relativistic hydrodynamics. // Teor. Mat. Fiz., 1979, vol. 40, No 3, p. 394-406 (in Russian).

71. S.Tomonaga. The newest development in quantum electrodynamics. // Progr. Theor. Phys., 1946, vol. 1, No 1, p. 27-32.

72. J.Schwinger. The newest development in quantum electrodynamics. Phys. Rev., 1948, vol. 74, No 10, p. 1439-1461, (+Phys. Rev., 1949, vol. 75, p. 651; +Phys. Rev., 1949, vol. 76 , p. 790).

73. N.N.Bogolubov, D.V.Shirkov. Introduction to the theory of quantized fields. Wiley, New York, 1980.

74. I.B.Khriplovich. Green's functions in theories with non-Abelian gauge group. // J. Nucl. Phys. (Sov.), 1969, vol. 10, No 2, p. 409-424.

75. U.Heinz. Kinetic theory for plasmas with non-Abelian interactions. // Phys. Rev. Lett., 1983, vol. 51, No 5, p. 351-354. 


\title{
Рівняння термопольової кінетики та гідродинаміки густих квантових ядерних систем
}

\author{
М.В.Токарчук ${ }^{1}$, Т.Арімітсу $^{2}$, О.Є.Кобрин ${ }^{1}$ \\ 1 Інститут фізики конденсованих систем НАН України, 290011 \\ м.Львів, вул. Свєнціцького, 1 \\ 2 Інститут фізики Університету м. Тсукуба, Ібаракі 305, Японія
}

Отримано 23 червня 1998 р.

Представлено основні рівняння нерівноважної термопольової динаміки густих квантових систем. Формулювання нерівноважної термопольової динаміки подано методом нерівноважного статистичного оператора Д.М.Зубарєва. Отримано рівняння гідродинаміки у термопольовому представленні. Розглянуто два рівні опису кінетики та гідродинаміки густої ядерної матерії: квантові системи сильно зв'язаних станів та кварк-глюонна плазма. Для цих систем отримано узагальнені рівняння переносу узгодженого опису кінетики та гідродинаміки. Розглянуто граничні випадки.

Ключові слова: нерівноважна термопольова динаміка, кінетика, гідродинаміка, кінетичні рівняння, коефіцієнти переносу, зв'язані стани, кварк-глюонна плазма

PACS: $12.38 . \mathrm{Mh}, 24.85 .+p, 52.25 . \mathrm{Dg}, 52.25 . \mathrm{Fi}, 82.20 . \mathrm{Mj}$ 